\title{
Uniform Asymptotic Expansions for the Discrete Chebyshev Polynomials
}

\author{
J. H. Pan and R. Wong ${ }^{\mathrm{b}}$ \\ ${ }^{a}$ Department of Mathematics, City University of Hong Kong, Tat Chee Avenue, Kowloon, \\ Hong Kong \\ ${ }^{b}$ Liu Bie Ju Centre for Mathematical Sciences, City University of Hong Kong, Tat Chee \\ Avenue, Kowloon, Hong Kong
}

\begin{abstract}
The discrete Chebyshev polynomials $t_{n}(x, N)$ are orthogonal with respect to a distribution function, which is a step function with jumps one unit at the points $x=0,1, \cdots, N-1, \mathrm{~N}$ being a fixed positive integer. By using a double integral representation, we derive two asymptotic expansions for $t_{n}(a N, N+1)$ in the double scaling limit, namely, $N \rightarrow \infty$ and $n / N \rightarrow b$, where $b \in(0,1)$ and $a \in(-\infty, \infty)$. One expansion involves the confluent hypergeometric function and holds uniformly for $a \in\left[0, \frac{1}{2}\right]$, and the other involves the Gamma function and holds uniformly for $a \in(-\infty, 0)$. Both intervals of validity of these two expansions can be extended slightly to include a neighborhood of the origin. Asymptotic expansions for $a \geq \frac{1}{2}$ can be obtained via a symmetry relation of $t_{n}(a N, N+1)$ with respect to $a=\frac{1}{2}$. Asymptotic formulas for small and large zeros of $t_{n}(x, N+1)$ are also given.
\end{abstract}




\section{INTRODUCTION}

In Szegö [1, p.33], the discrete Chebyshev polynomials are defined by

$$
t_{n}(x, N)=n ! \triangle^{n}\left(\begin{array}{l}
x \\
n
\end{array}\right)\left(\begin{array}{c}
x-N \\
n
\end{array}\right), \quad n=0,1,2, \cdots, N-1,
$$

where $\triangle$ denotes the difference operator

$$
\begin{aligned}
\triangle f(x) & =f(x+1)-f(x), \\
\triangle^{n} f(x) & =\triangle\left\{\triangle^{n-1} f(x)\right\} \\
& =f(x+n)-\left(\begin{array}{l}
n \\
1
\end{array}\right) f(x+n-1)+\cdots+(-1)^{n} f(x) .
\end{aligned}
$$

These polynomials are orthogonal with respect to the distribution $\mathrm{d} \alpha(x)$ of Stieltjes type, where $\alpha(x)$ is a step function with jumps one unit at the points $x=0,1, \cdots, N-1, N$ being a fixed positive integer. More precisely, we have

$$
\int_{-\infty}^{\infty} t_{n}(x, N) t_{m}(x, N) \mathrm{d} \alpha(x)=\sum_{x=0,1, \cdots, N-1} t_{n}(x, N) t_{m}(x, N)=0
$$

if $n \neq m$, and

$$
\begin{aligned}
\int_{-\infty}^{\infty}\left\{t_{n}(x, N)\right\}^{2} \mathrm{~d} \alpha(x) & =\sum_{x=0,1, \cdots, N-1}\left\{t_{n}(x, N)\right\}^{2} \\
& =\frac{N\left(N^{2}-1^{2}\right)\left(N^{2}-2^{2}\right) \cdots\left(N^{2}-n^{2}\right)}{2 n+1}
\end{aligned}
$$

for $n, m=0,1,2, \cdots, N-1$. The last two formulas hold for all non-negative values of $n$ and $m$; in fact, they are trivial for $n \geq N$ or $m \geq N$, since $t_{n}(x, N)=0$ for $x=0,1,2, \cdots, N-1$, if $n \geq N$.

The discrete Chebyshev polynomials can also be defined as a special case of Hahn polynomials

$$
\begin{aligned}
Q_{n}(x ; \alpha, \beta, N) & ={ }_{3} F_{2}(-n,-x, n+\alpha+\beta+1 ;-N, \alpha+1 ; 1) \\
& =\sum_{k=0}^{n} \frac{(-n)_{k}(-x)_{k}(n+\alpha+\beta+1)_{k}}{(-N)_{k}(\alpha+1)_{k} k !}
\end{aligned}
$$

and we have

$$
\begin{aligned}
t_{n}(x, N) & =(-1)^{n}(N-n)_{n} Q_{n}(x ; 0,0, N-1) \\
& =(-1)^{n}(N-n)_{n} \sum_{k=0}^{n} \frac{(-n)_{k}(-x)_{k}(n+1)_{k}}{(-N+1)_{k} k ! k !}
\end{aligned}
$$

see [2, p.174 and 176] and [3]. These polynomials are used in least squares polynomial approximation [4, p.350] and sequential smoothing of numerical data [5]. (In 44, they are referred to as Gram polynomials; see Chihara [3, p.162].) 
In this paper, we are concerned with the problem of finding asymptotic expansions for the polynomials $t_{n}(x, N)$. Already, a tremendous amount of research has been carried out on the asymptotics of various discrete orthogonal polynomials. For instance, we have [6] and [7] for Charlier polynomials, [8] and [9] for Meixner polynomials, [10] and [11] for Krawtchouk polynomials, and [12] and [13] for Tricomi-Carlitz polynomials. There is also an important research monograph by Baik et al. [14 on a general asymptotic method, based on the Riemann-Hilbert approach, which in principle can be applied to all discrete orthogonal polynomials. However, their result is so general that it does not provide explicit asymptotic formulas for specific polynomials. Strangely enough, for the discrete Chebyshev polynomials there does not seem to be any asymptotic result in the literature; at least, we have not been able to find any, and the purpose of this paper is to fill in this gap.

For convenience, we shall consider the behavior of $t_{n}(x, N+1)$ instead of $t_{n}(x, N)$. Since the Hahn polynomials satisfy the equation [15, (1.15)]

$$
Q_{n}(N-x ; \alpha, \beta, N)=\frac{Q_{n}(x ; \alpha, \beta, N)}{Q_{n}(N ; \alpha, \beta, N)}
$$

and $Q_{n}(N ; 0,0, N)=(-1)^{n}$ (note that there are slight differences in the definition of $Q_{n}$ [15, (1.1)]), the discrete Chebyshev polynomials enjoy the symmetry relation

$$
t_{n}(x, N+1)=(-1)^{n} t_{n}(N-x, N+1) .
$$

In view of (1.8), we may restrict $x$ to the interval $-\infty<x \leq \frac{1}{2} N$. We are interested in the behavior of $t_{n}(x, N+1)$ as $n, N \rightarrow \infty$ in such a way that the ratios

$$
a=x / N \quad \text { and } \quad b=n / N
$$

satisfy the inequalities

$$
-\infty<a \leq \frac{1}{2} \quad \text { and } \quad 0<b<1
$$

As a special case of our general result, we will show that for fixed value of $x \in[0, \infty)$, there exists a number $\eta<0$ such that

$$
\begin{aligned}
t_{n}(x, N+1)= & \frac{(-1)^{n+1} \Gamma(n+N+2) N^{x} n^{-2 x-2} \Gamma(x+1)}{\Gamma(N+1) \pi} \\
& \times\left\{\sin \pi x\left[1+O\left(\frac{1}{N}\right)\right]+O\left(e^{\eta N}\right)\right\} .
\end{aligned}
$$

This result also holds when $x$ is negative, except that the term $O\left(e^{\eta N}\right)$ is absent.

The arrangement of the present paper is as follows. In $₫ 2$, we present two (2-dimensional) integral representations for $t_{n}(x, N+1)$, one for the case $x<0$ and the other for $x \geq 0$. In \$3, we transform the integration variables $(w, t)$ to two new variables $(u, \tau)$, and deduce the double integrals to their canonical forms, from which uniform asymptotic expansions can be derived by a standard integration-by-parts procedure. In \$4, we prove that the transformation $(w, t) \rightarrow(u, \tau)$ is one-to-one and analytic. In \$5, we investigate the analyticity of the integrands of the double integrals in (3.20) and (3.29). The desired asymptotic 
expansions are then derived in $₫ \underline{6}$, and the estimation of the error terms is done in $\$ 7$, In \$8, we state the final results and give a list of special cases; and we extend the regions of validity of the final results in $\$ 9$. In $\$ 10$, we present some asymptotic formulas for the large and small zeros of the polynomial under consideration.

\section{INTEGRAL REPRESENTATION}

From (1.6), we have

$$
\begin{aligned}
t_{n}(x, N+1) & =(-1)^{n}(N+1-n)_{n} Q_{n}(x ; 0,0, N) \\
& =(-1)^{n}(N+1-n)_{n} \sum_{k=0}^{n} \frac{(-n)_{k}(-x)_{k}(n+1)_{k}}{(-N)_{k} k ! k !} .
\end{aligned}
$$

Using beta integral, we also have

$$
\begin{aligned}
\frac{(n+1)_{k}}{(-N)_{k}} & =(-1)^{k} \frac{\Gamma(n+1+k) \Gamma(N+1-k)}{\Gamma(n+1) \Gamma(N+1)} \\
& =(-1)^{k} \frac{\Gamma(n+N+2)}{\Gamma(n+1) \Gamma(N+1)} \int_{0}^{1}(1-t)^{n+k} t^{N-k} \mathrm{dt}
\end{aligned}
$$

for $0 \leq k \leq n \leq N$. Substituting (2.2) in (2.1) and interchanging the summation and integration signs, we obtain

$$
\begin{aligned}
t_{n}(x, N+1)= & (-1)^{n} \frac{\Gamma(n+N+2)}{\Gamma(n+1) \Gamma(N+1-n)} \\
& \times \int_{0}^{1}(1-t)^{n} t^{N}{ }_{2} F_{1}\left(-n,-x ; 1 ; 1-t^{-1}\right) \mathrm{d} t .
\end{aligned}
$$

In the above integral, we now use the new scales $a=x / N$ and $b=n / N$ introduced in (1.9), and consider separate cases (i) $0 \leq a \leq \frac{1}{2}$ and (ii) $a<0$; cf. (1.10).

Recall the identity [16, (15.8.1)]

$$
{ }_{2} F_{1}\left(-n,-x ; 1 ; 1-t^{-1}\right)=t^{-n}{ }_{2} F_{1}(-n, 1+x ; 1 ; 1-t)
$$

and the contour integral representation [2, p.287]

$$
{ }_{2} F_{1}(a, b ; c ; z)=\frac{\Gamma(c) \Gamma(1+b-c)}{2 \pi i \Gamma(b)} \int_{\gamma_{1}} w^{b-1}(w-1)^{c-b-1}(1-w z)^{-a} \mathrm{~d} w
$$

where $\operatorname{Re} b>0$ and the curve $\gamma_{1}$ starts at $w=0$, runs along the lower edge of the positive real line towards $w=1$, encircles the point $w=1$ in the counterclockwise direction, and returns to the origin along the upper edge of the positive real line. For case (i) $0 \leq a \leq \frac{1}{2}$, we apply (2.4) and (2.5) to (2.3), and obtain the double integral representation

$$
t_{n}(x, N+1)=\frac{(-1)^{n}}{2 \pi i} \frac{\Gamma(n+N+2)}{\Gamma(n+1) \Gamma(N-n+1)} \int_{0}^{1} \int_{\gamma_{1}} \frac{1}{w-1} e^{N f(t, w)} \mathrm{d} w \mathrm{~d} t,
$$


where

$$
f(t, w)=b \ln (1-t)+(1-b) \ln t+a \ln w-a \ln (w-1)+b \ln [1-(1-t) w]
$$

The function $f(t, w)$ has a cut $(-\infty, 1]$ in the $w$-plane. Since the coefficients of $\ln t$, $\ln (1-t)$ and $\ln [1-(1-t) w]$ in $N f(t, w)$ are all nonnegative integers, there is no need to have cuts in the $t$-plane for $N f(t, w)$.

For case (ii), we use instead of (2.5) the contour integral representation

$$
{ }_{2} F_{1}(a, b ; c ; z)=\frac{-\Gamma(c) \Gamma(1-b)}{2 \pi i \Gamma(c-b)} \int_{\gamma_{2}}(-w)^{b-1}(1-w)^{c-b-1}(1-w z)^{-a} \mathrm{~d} w,
$$

where $\operatorname{Re} c>\operatorname{Re} b$ and the curve $\gamma_{2}$ starts at $w=1$, moves along the upper edge of the positive real line towards $w=0$, encircles the origin in the counterclockwise direction, and returns to $w=1$ along the lower edge of the positive real line. (A proof of this formula can be given along the same lines as that of (2.5) $)$.) Thus, we have

$$
t_{n}(x, N+1)=\frac{(-1)^{n}}{2 \pi i} \frac{\Gamma(n+N+2)}{\Gamma(n+1) \Gamma(N-n+1)} \int_{0}^{1} \int_{\gamma_{2}} \frac{1}{w-1} e^{N \widetilde{f}(t, w)} \mathrm{d} w \mathrm{~d} t
$$

where

$$
\widetilde{f}(t, w)=b \ln (1-t)+(1-b) \ln t+a \ln (-w)-a \ln (1-w)+b \ln [1-(1-t) w] .
$$

Similar to $f(t, w)$ in (2.7), the function $\tilde{f}(t, w)$ has a cut $[0,+\infty)$ in the $w$-plane and no cut in the $t$-plane.

\section{REDUCTION TO CANONICAL INTEGRALS}

To obtain large- $\lambda$ behavior of higher dimensional integral

$$
I(\lambda)=\int_{\gamma} f(z) e^{\lambda S(z)} \mathrm{d} z, \quad z=\left(z_{1}, z_{2}, \cdots, z_{n}\right) \in \mathbb{C}^{n},
$$

where $\mathrm{d} z=\mathrm{d} z_{1} \cdots \mathrm{d} z_{n}$ and $\gamma$ is a smooth manifold of (real) dimension $n$, Fdeoryuk [17] showed that just like the classical method of steepest descent, the saddle points of the phase function $S(z)$ (i.e., the zeros of the first-order derivatives of $S(z)$ ) play an important role; see also Kaminski [18]. So, let us first investigate the location of the saddle points of the phase functions $f(t, w)$ and $\widetilde{f}(t, w)$ in (2.7) and (2.10).

Upon solving the equations

$$
\frac{\partial f}{\partial t}(t, w)=0 \quad \text { and } \quad \frac{\partial f}{\partial w}(t, w)=0
$$

and the equations

$$
\frac{\partial \tilde{f}}{\partial t}(t, w)=0 \quad \text { and } \quad \frac{\partial \tilde{f}}{\partial w}(t, w)=0
$$


one finds that the phase functions $f(t, w)$ and $\tilde{f}(t, w)$ have the same two sets of saddle points

$$
t_{+}=\frac{2-2 a-b^{2}+b \sqrt{b^{2}-4 a+4 a^{2}}}{2(1-a)(1+b)}, \quad w_{+}=\frac{b+\sqrt{b^{2}-4 a+4 a^{2}}}{2 b}
$$

and

$$
t_{-}=\frac{2-2 a-b^{2}-b \sqrt{b^{2}-4 a+4 a^{2}}}{2(1-a)(1+b)}, \quad w_{-}=\frac{b-\sqrt{b^{2}-4 a+4 a^{2}}}{2 b}
$$

Note that $w_{+}$and $w_{-}$, as well as $t_{+}$and $t_{-}$, coalesce when $a$ approaches $a_{+}$and $a_{-}$, where

$$
a_{ \pm}=\frac{1 \pm \sqrt{1-b^{2}}}{2}
$$

The following facts are easily verified:

1. For $a<0, w_{ \pm}$are both real and $w_{-}<0<1<w_{+}$. As $a \rightarrow 0, w_{-} \rightarrow 0^{-}$and $w_{+} \rightarrow 1^{+}$.

2. For $0<a<a_{-}$, both $w_{+}$and $w_{-}$are real and $0<w_{-}<\frac{1}{2}<w_{+}<1$. As $a \rightarrow a_{-}$, both $w_{+}$and $w_{-}$approach $\frac{1}{2}$.

3. For $a_{-}<a<a_{+}, w_{ \pm}$are complex conjugates, the real parts of which are both equal to $\frac{1}{2}$. At $a=\frac{1}{2}, w_{ \pm}$attain their highest and lowest point on the line $\operatorname{Re} w=\frac{1}{2}$.

4. The locations of $w_{ \pm}$in the cases $a_{+}<a<1$ and $a>1$ are similar to those in the cases $0<a<a_{-}$and $a<0$, respectively. This fact also reflects in the symmetry property of the discrete Chebyshev polynomial $t_{n}(a N, N+1)$ in $a$ with respect to $a=\frac{1}{2} ;$ cf. (1.8) and (1.10).

Figure 1 summarizes the movements of $w_{ \pm}$.

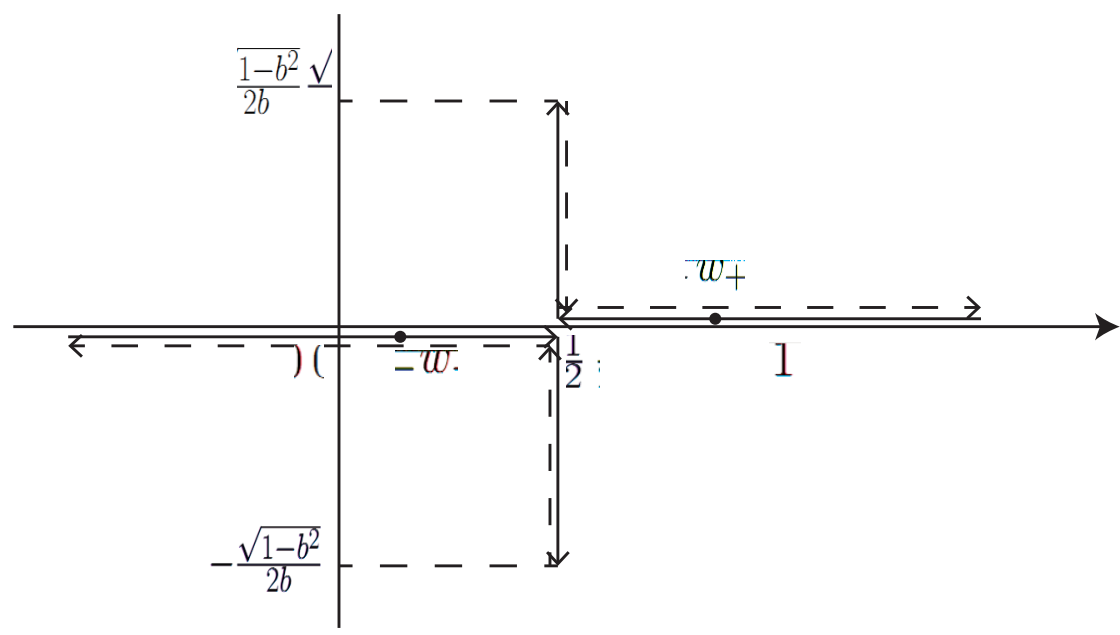

Figure 1: Movements of $w_{ \pm}$. 
In view of (1.10), we only need consider the case $-\infty<a \leq \frac{1}{2}$. We again divide our discussion into two subcases: (i) $0 \leq a \leq \frac{1}{2}$ and (ii) $-\infty<a<0$.

Case (i) $0 \leq a \leq \frac{1}{2}$

Returning to the integral representation (2.6), we now show that this double integral can be reduced to a canonical form, and that the original integration surface $[0,1] \times \gamma_{1}$ in the $t \times w$ plane can be deformed into a new integration surface. An outline of the procedure is as follows. First, for each fix $w \in \gamma_{1}$, we find a steepest descent path of the variable $t$, which passes through a relevant saddle point $t_{0}(w)$ depending on $w$. We denote this path by $\rho_{t}$. Note that not only the saddle point $t_{0}(w)$, but all points $t$ on the path $\rho_{t}$ depend on $w$. Thus, the phase function $f\left(t_{0}(w), w\right)$ is now a function of $w$ alone. Second, deform the integration path $\gamma_{1}$ in (2.6) into a steepest descent path of $w$, passing through $w_{ \pm}$given in (3.1) and (3.2). We denote this path by $\gamma_{w}$. It will be shown that these saddle points are indeed saddle points of $f\left(t_{0}(w), w\right)$.

To find the relevant saddle point $t_{0}(w)$, we solve the equation

$$
\frac{\partial}{\partial t} f(t, w)=0
$$

and obtain

$$
t_{0}^{ \pm}(w)=\frac{2 w-1 \pm \sqrt{1+4 b^{2}(w-1) w}}{2(1+b) w}
$$

Here, we have introduced two more cuts in the $w$-plane, both on the vertical line Re $w=\frac{1}{2}$, one from $\left(b+i \sqrt{1-b^{2}}\right) / 2 b$ all the way to $w=\frac{1}{2}+i \infty$, and the other from $\left(b-i \sqrt{1-b^{2}}\right) / 2 b$ all the way to $w=\frac{1}{2}-i \infty$. As remarked earlier, the two saddle points $\left(t_{+}, w_{+}\right)$and $\left(t_{-}, w_{-}\right)$in (3.1) and (3.2) play an important role in determining the asymptotic behavior of $t_{n}(x, N+1)$. So, we must be careful in choosing the right saddle point from the two given in (3.4). For $a \leq \frac{1}{2}$, it can be shown that $t_{0}^{+}\left(w_{ \pm}\right)=t_{ \pm}$. Thus, $t_{0}^{+}(w)$ is our relevant saddle point. Throughout the following discussion, we shall simply write $t_{0}(w)$ for $t_{0}^{+}(w)$, when no misunderstanding would arise.

As we have explained previously, for fixed $w$ in the whole $w$-plane and $b \in(0,1)$, the function $e^{N f(t, w)}$ inside the integral in (2.6) has no singularity in the $t$-plane. However, to make it possible for us to deform the original integration path $[0,1]$ into a steepest descent path $\rho_{t}$, we have to define the function $f(t, w)$ in such a way that it avoids the cut $(-\infty, 1]$ in the $w$-plane, and three additional cuts in the $t$-plane, $(-\infty, 0],[1, \infty)$ and also the radial line starting from $1-1 / w$ and satisfying $1-(1-t) w<0$. With this in mind, we now define the steepest descent path $\rho_{t}$, passing through $t_{0}(w)$, by

$$
\begin{aligned}
& \operatorname{Im} f(t, w)=\operatorname{Im} f\left(t_{0}(w), w\right), \\
& \operatorname{Re} f(t, w) \leq \operatorname{Re} f\left(t_{0}(w), w\right)
\end{aligned}
$$

see Figure 2 and Figure 3, It is now standard to define a transformation $t \rightarrow \tau$ by

$$
\tau^{2}=f\left(t_{0}(w), w\right)-f(t, w)
$$




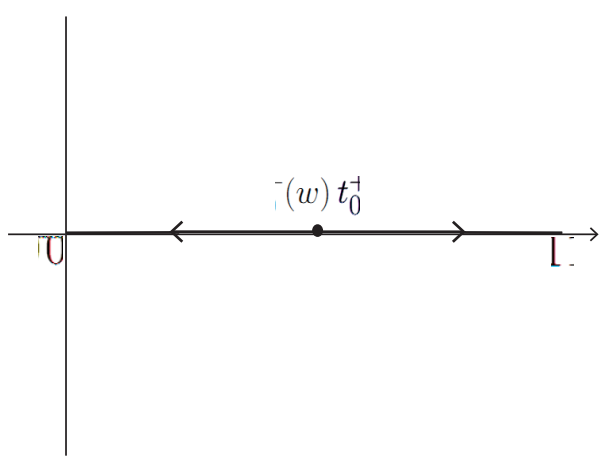

Figure 2: Steepest descent path in the $t$-plane when $w$ is real.

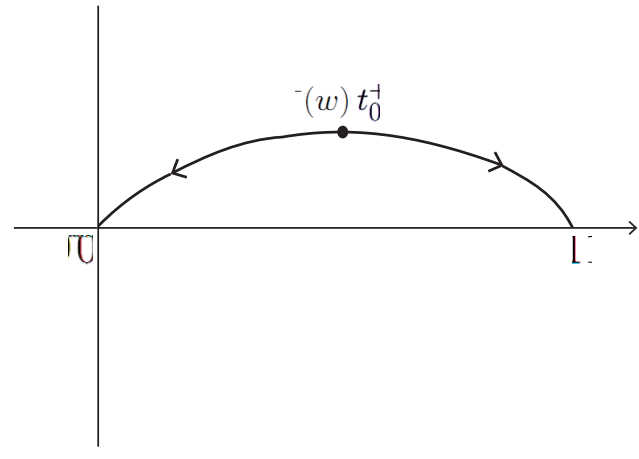

Figure 3: Steepest descent path in the $t$-plane when $w$ is complex; e.g., $w=$ $0.5+0.2 i$.

Note that we have $\tau=-\infty$ when $t=0$, and $\tau=+\infty$ when $t=1$. Furthermore, this mapping is one-to-one with $\tau$ running from $-\infty$ to $\infty$, and takes $t=t_{0}(w)$ to $\tau=0$. From (2.7) and (3.6), we have

$$
\begin{array}{ll}
\frac{d t}{d \tau}=\frac{2 \tau}{-\partial f(t, w) / \partial t}=\frac{2 \tau t(1-t)[1-(1-t) w]}{(1+b) w\left(t-t_{0}^{+}(w)\right)\left(t-t_{0}^{-}(w)\right)}, & \tau \neq 0, \\
\frac{d t}{d \tau}=\left\{\frac{2 t_{0}^{+}(w)\left(1-t_{0}^{+}(w)\right)\left[1-\left(1-t_{0}^{+}(w)\right) w\right]}{\sqrt{1+4 b^{2}(w-1) w}}\right\}^{1 / 2}, & \tau=0 .
\end{array}
$$

Coupling (2.6) and (3.6) gives

$$
\begin{aligned}
t_{n}(x, N+1)= & \frac{(-1)^{n}}{2 \pi i} \frac{\Gamma(n+N+2)}{\Gamma(n+1) \Gamma(N-n+1)} \\
& \times \int_{-\infty}^{+\infty} \int_{\gamma_{1}} \frac{1}{w-1} e^{N f\left(t_{0}(w), w\right)} e^{-N \tau^{2}} \frac{\mathrm{d} t}{\mathrm{~d} \tau} \mathrm{d} w \mathrm{~d} \tau,
\end{aligned}
$$

where $\gamma_{1}$ is the integration path of $w$ in (2.6). Note that the first variable of $f$ in (3.8) is now $t_{0}(w)$, instead of $t$. So the phase function depends only on $w$. Setting $\partial f\left(t_{0}(w), w\right) / \partial w=0$, we obtain saddle points

$$
w_{ \pm}=\frac{b \pm \sqrt{b^{2}-4 a+4 a^{2}}}{2 b} ;
$$

cf. (3.1) and (3.2). We now deform $\gamma_{1}$ into steepest descent paths passing through $w_{+}$and $w_{-}$. Recall that the movements of $w_{ \pm}$are summerized in Figure 1, as $a$ runs along the real line. In the case $a_{-}<a \leq \frac{1}{2}$, the saddle points $w_{ \pm}$are complex conjugate numbers with real part equal to $\frac{1}{2}$. The steepest descent path passing through $w_{+}$is given by

$$
\begin{aligned}
& \operatorname{Im} f\left(t_{0}(w), w\right)=\operatorname{Im} f\left(t_{0}\left(w_{+}\right), w_{+}\right), \\
& \operatorname{Re} f\left(t_{0}(w), w\right) \leq \operatorname{Re} f\left(t_{0}\left(w_{+}\right), w_{+}\right) .
\end{aligned}
$$

The steepest descent path through $w_{-}$can be obtained by using the symmetry property with respect to the real axis; see Figure 4. 


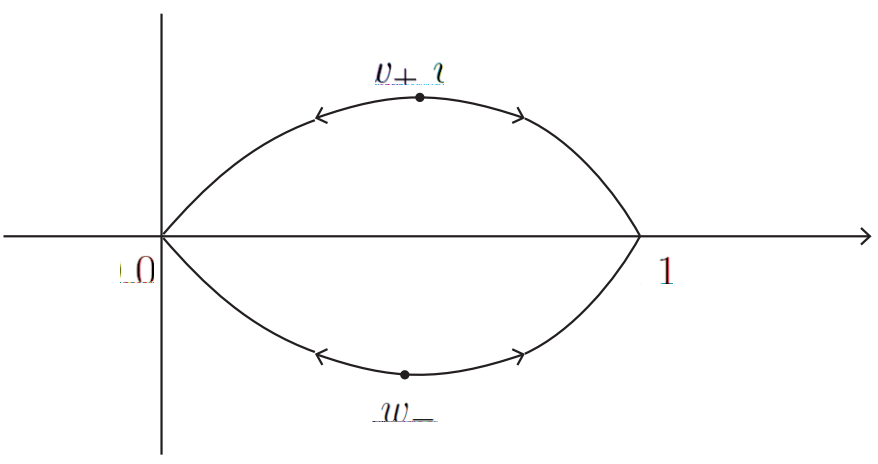

Figure 4: Steepest descent path of $w$ when $a_{-}<a \leq \frac{1}{2}$.

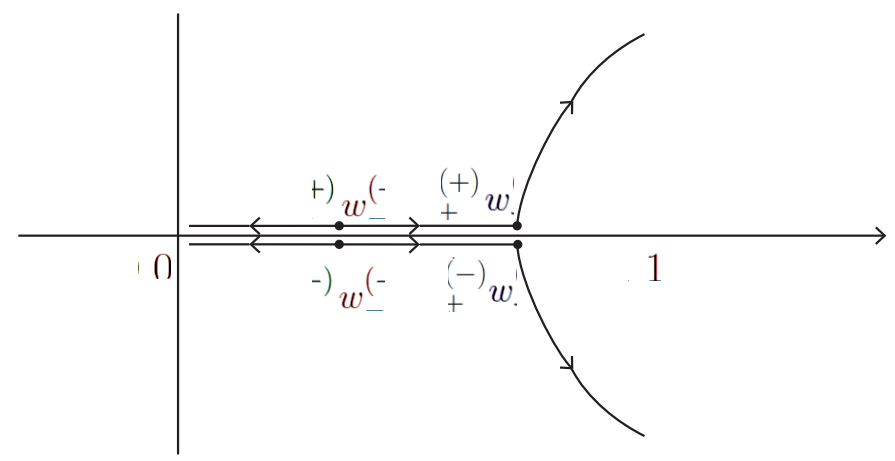

Figure 5: Steepest descent path of $w$ when $0 \leq a<a_{-}$.

When $0 \leq a<a_{-}$, both $w_{ \pm}$are real and lie in the interval [0,1]; thus, $w_{ \pm}$appear on both upper and lower edges of the cut along the real line. We denote these points by $w_{ \pm}^{(+)}$and $w_{ \pm}^{(-)}$; see Figure [5. Since $\operatorname{Re} f\left(t_{0}\left(w_{-}^{( \pm)}\right), w_{-}^{( \pm)}\right)>\operatorname{Re} f\left(t_{0}\left(w_{+}^{( \pm)}\right), w_{+}^{( \pm)}\right), w_{-}^{( \pm)}$is a local maximum and the steepest descent path through $w_{-}^{( \pm)}$is along the real axis. From Figure 1, it is clear that this path is on the real interval $\left(0, w_{+}^{( \pm)}\right)$. The steepest descent path throught $w_{+}^{( \pm)}$is the curve perpendicular to the real axis; cf. Figure 5 .

Motivated by the work of Jin and Wong [8], the movements of the saddle points $w_{ \pm}$ and the steepest descent paths passing through them suggest that we should compare the integral of $w$ in (3.8) with the confluent hypergeometric function [16, p.326, (13.4.9)]

$$
M(d, c, z)=\frac{\Gamma(c) \Gamma(1+d-c)}{2 \pi i \Gamma(d)} \int_{\gamma_{1}} u^{d-1}(u-1)^{c-d-1} e^{z u} \mathrm{~d} u,
$$

where Re $d>0$ and the integration curve $\gamma_{1}$ is the same one as that given in (2.5) or (3.8). Since $M(d, c, z)$ is a meromorphic function of $c$ with poles at $0,-1,-2, \cdots$, the function $\mathbf{M}(d, c, z):=M(d, c, z) / \Gamma(c)$ is entire in both $d$ and $c$; see [19, p.255]. With $d=a N+1$ $\left(0 \leq a \leq \frac{1}{2}\right), c=1, z=\eta N$, (3.11) gives

$$
\mathbf{M}(a N+1,1, \eta N)=\frac{1}{2 \pi i} \int_{\gamma_{1}} e^{N \psi(u)} \frac{\mathrm{d} u}{u-1}
$$

where

$$
\psi(u)=a \ln u-a \ln (u-1)+\eta u
$$




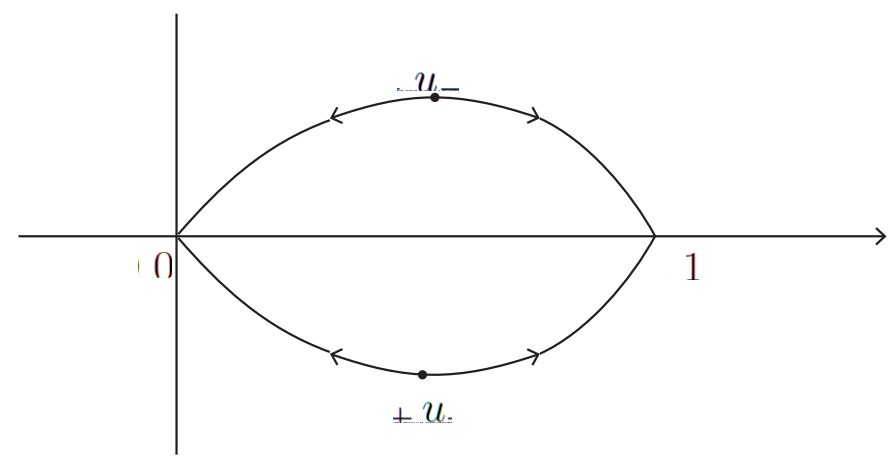

Figure 6: Steepest descent path of $u$ when $a>0$ and $-4 a<\eta<0$.

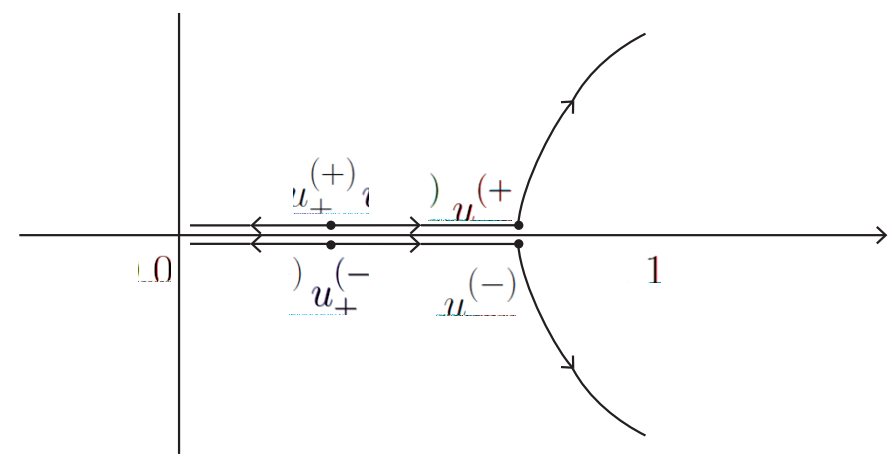

Figure 7: Steepest descent path of $u$ when $a>0$ and $\eta<-4 a$.

and the saddle points of $\psi$ are given by

$$
u_{ \pm}=\frac{\eta \pm \sqrt{\eta^{2}+4 a \eta}}{2 \eta} .
$$

The shape of the steepest descent paths of $\psi$ depends on the sign of $a$ and the value of $\eta$. Here, we are concerned with only the two cases: (i) $a \geq 0$ and $-4 a<\eta<0$, (ii) $a \geq 0$ and $\eta<-4 a$; see Figures 6 and $[7$.

We also observe that when $a \geq 0$, the movements of $u_{ \pm}$are like those of $w_{\mp}$, with the corresponding subcases

$$
\begin{aligned}
& a_{-}<a<\frac{1}{2} \quad \leftrightarrow \quad-4 a<\eta<0 \\
& 0 \leq a \leq a_{-} \quad \leftrightarrow \quad \eta \leq-4 a
\end{aligned}
$$

Thus, we define the mapping $w \rightarrow u$ by

$$
\begin{aligned}
f\left(t_{0}(w), w\right) & =\psi(u)+\gamma \\
& =a \ln u-a \ln (u-1)+\eta u+\gamma
\end{aligned}
$$

with

$$
u\left(w_{+}\right)=u_{-}, \quad u\left(w_{-}\right)=u_{+},
$$

where $\eta$ and $\gamma$ are real numbers. Note that since $w_{ \pm}$are on both edges of the real line (similarly, $u_{ \pm}$are also on both edges of the real line) when $0 \leq a \leq a_{-}$, (3.16) should be 
understood in the sense:

$$
\begin{array}{ll}
u\left(w_{+}^{(+)}\right)=u_{-}^{(+)}, & u\left(w_{-}^{(+)}\right)=u_{+}^{(+)}, \\
u\left(w_{+}^{(-)}\right)=u_{-}^{(-)}, & u\left(w_{-}^{(-)}\right)=u_{+}^{(-)} .
\end{array}
$$

It can be easily verified that (3.17a) and (3.17b) are equivalent in the case $0 \leq a \leq a_{-}$. The existence and uniqueness of the numbers $\eta$ and $\gamma$ as well as the one-to-one and analytic property of the mapping $w \rightarrow u$ defined in (3.15) will be established in the following section.

Note that we have chosen the coefficients of $\ln u$ and $\ln (u-1)$ in $\psi(u)$ to be $a$. The reason is that with this choice, the local expansions of $f\left(t_{0}(w), w\right)$ at $w=0$ and $w=1$ and that of $\psi(u)+\gamma$ at the corresponding points $u=0$ and $u=1$ are the same.

From (3.15), we have

$$
\begin{aligned}
\frac{\mathrm{d} w}{\mathrm{~d} u} & =\frac{\mathrm{d} \psi}{\mathrm{d} u} / \frac{\mathrm{d} f\left(t_{0}(w), w\right)}{\mathrm{d} w} \\
& =\frac{\eta\left(u-u_{+}\right)\left(u-u_{-}\right) w(1-w)\left[(2 a-1)-\sqrt{1+4 b^{2} w^{2}-4 b^{2} w}\right]}{-2 b^{2} u(u-1)\left(w-w_{+}\right)\left(w-w_{-}\right)}
\end{aligned}
$$

for $u \neq u_{ \pm}$, and by l'Hôpital's rule,

$$
\frac{\mathrm{d} w}{\mathrm{~d} u}=\left[\frac{\sqrt{\eta^{2}+4 a \eta}(1-2 a)(1-a)(-\eta)}{b^{3} \sqrt{b^{2}-4 a+4 a^{2}}}\right]^{1 / 2}
$$

for $u=u_{ \pm}$. (Note that $\left.\sqrt{1+4 b^{2} w_{\mp}^{2}-4 b^{2} w_{\mp}}=1-2 a\right)$.

By coupling (3.8) and (3.15), the double integral representation of $t_{n}(x, N+1)$ given in (2.6) is now reduced to the canonical form

$$
\begin{aligned}
t_{n}(x, N+1)=\frac{(-1)^{n}}{2 \pi i} & \frac{\Gamma(n+N+2)}{\Gamma(n+1) \Gamma(N-n+1)} e^{N \gamma} \\
& \times \int_{-\infty}^{+\infty} \int_{\gamma_{u}} \frac{h(u, \tau)}{u-1} e^{N \psi(u)-N \tau^{2}} \mathrm{~d} u \mathrm{~d} \tau,
\end{aligned}
$$

where

$$
h(u, \tau)=\frac{u-1}{w-1} \frac{\mathrm{d} w}{\mathrm{~d} u} \frac{\mathrm{d} t}{\mathrm{~d} \tau}
$$

and $\gamma_{u}$ is the steepest descent path depicted in Figures 6 and 7 when $a_{-}<a \leq \frac{1}{2}$ and $0 \leq a<a_{-}$, respectively.

Before concluding our discussion of Case (i): $0 \leq a \leq \frac{1}{2}$, let us give a summary of the reduction process from (2.6) to (3.20). We first deformed the original surface $[0,1] \times \gamma_{1} \in \mathbb{C}^{2}$ in (2.6) into $\rho_{t} \times \gamma_{w} \in \mathbb{C}^{2}$, where $\rho_{t}$ is the steepest descent path in the $t$-plane of the phase function $f(t, w)$ in (2.6), which passes through the saddle point 
$t_{0}(w)=t_{0}^{+}(w)$ in (3.4) for each fixed $w$, and where $\gamma_{w}$ is the steepest descent path of $f\left(t_{0}(w), w\right)$ in the $w$-plane, which passes through the saddle points $w_{ \pm}$given in (3.9). Next, for every fixed $w$, we introduced a one-to-one analytic map $t \rightarrow \tau$, defined by (3.6), which maps $\rho_{t}$ to $(-\infty,+\infty)$, and a map $w \rightarrow u$, defined by (3.15), which maps $\gamma_{w} \rightarrow \gamma_{u}$, where $\gamma_{u}$ is the steepest descent path in the $u$-plane for the canonical integral (3.20). In the sense of Kaminski [18], we may refer to $S_{\tau \times u}:=(-\infty, \infty) \times \gamma_{u}$ as a steepest descent surface in $\mathbb{C}^{2}$. Thus, the 2-dimensional transformation $(t, w) \rightarrow(\tau, u)$ defined by

$$
\begin{aligned}
b \ln (1-t)+(1-b) \ln t & +a \ln w-a \ln (w-1)+b \ln [1-(1-t) w] \\
& =-\tau^{2}+a \ln u-a \ln (u-1)+\eta u+\gamma
\end{aligned}
$$

is, in fact, given in two stages; see (2.7), (3.6) and (3.15). This mapping $(t, w) \rightarrow(\tau, u)$ takes $\rho_{t} \times \gamma_{w}$ in the $\mathbb{C}^{2}$-plane $(t, w)$ into a steepest descent surface $S_{\tau \times u}$ in the $\mathbb{C}^{2}$-plane $(\tau, u)$. Hence, in conclusion, we have made the following change of surfaces of integration

$$
[0,1] \times \gamma_{1} \in \mathcal{C}_{t \times w}^{2} \quad \longrightarrow \quad \rho_{t} \times \gamma_{w} \in \mathcal{C}_{t \times w}^{2} \quad \longrightarrow \quad S_{\tau \times u} \in \mathcal{C}_{\tau \times u}^{2}
$$

Case (ii) $-\infty<a<0$.

In this case, instead of (2.6), we use the double integral representation (2.9). The argument here is similar to that in Case (i). But, we note from $\$ 2$ that when $a$ is negative, both $w_{-}$and $w_{+}$are real and $w_{-}<0<1<w_{+}$. Taking account of the shape of the contour $\gamma_{2}$ in (2.9), we need be concerned with only the saddle point $w_{-}$, and not $w_{+}$.

Since $a$ is negative and hence less than $\frac{1}{2}$, the relevant saddle point is again $t_{0}(w)=$ $t_{0}^{+}(w)$; see the argument following (3.4), except with $f$ replaced by $\tilde{f}$. Thus, (2.9) can be written as

$$
\begin{aligned}
t_{n}(x, N+1)= & \frac{(-1)^{n}}{2 \pi i} \frac{\Gamma(n+N+2)}{\Gamma(n+1) \Gamma(N-n+1)} \\
& \times \int_{-\infty}^{+\infty} \int_{\gamma_{2}} \frac{1}{w-1} e^{N \cdot \widetilde{f}\left(t_{0}(w), w\right)} e^{-N \tau^{2}} \frac{\mathrm{d} t}{\mathrm{~d} \tau} \mathrm{d} w \mathrm{~d} \tau
\end{aligned}
$$

where $\gamma_{2}$ is the integration path of $w$ in (2.9); cf. (3.8). However, the mapping $w \rightarrow u$ differs from that in Case (i). It can be shown that the second derivative of the phase function $\tilde{f}\left(t_{0}(w), w\right)$ is positive at $w=w_{-}$, and the steepest descent path passing through $w_{-}$is perpendicular to the real axis; see Figure 8 .

Recall the Hankel integral for the Gamma function

$$
\frac{1}{\Gamma(z)}=\frac{1}{2 \pi i} \int_{-\infty}^{(0+)} e^{u} u^{-z} \mathrm{~d} u
$$

where the contour is a loop starting at $-\infty$, encircling the origin in the counterclockwise direction, and returning to $-\infty$. With $z=-a N+1$ and $u$ replaced by $-u$, we obtain

$$
\frac{1}{\Gamma(-a N+1)}=\frac{N^{a N}}{2 \pi i} \int_{\infty}^{(0+)} \frac{1}{u} e^{N \widetilde{\psi}(u)} \mathrm{d} u,
$$




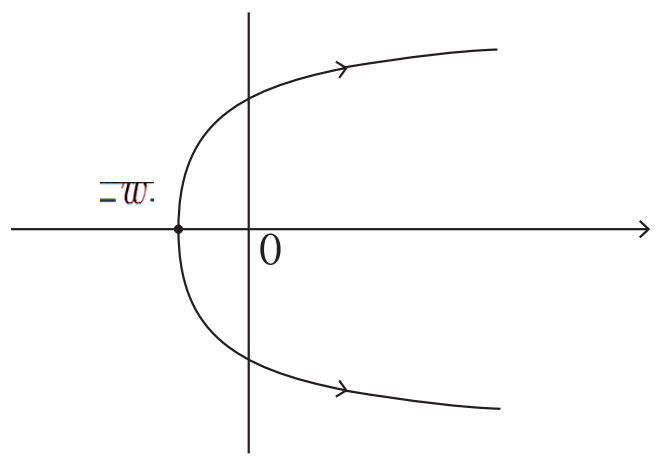

Figure 8: The steepest descent path of $w$, when $a<0$.

where

$$
\widetilde{\psi}(u)=a \ln (-u)-u,
$$

and the saddle point of $\widetilde{\psi}$ is at $u=a$.

Observe the similarities of the saddle points and the integration paths (see Figure 8) in the two integrals (3.23) and (3.25). Make the transformation $w \rightarrow u$ defined by

$$
\begin{aligned}
\widetilde{f}\left(t_{0}(w), w\right) & =\widetilde{\psi}(u)+\gamma \\
& =a \ln (-u)-u+\gamma
\end{aligned}
$$

with

$$
u\left(w_{-}\right)=a,
$$

where $\gamma$ is a constant to be determined.

Coupling (3.23) and (3.27) gives

$$
\begin{aligned}
t_{n}(x, N+1)= & \frac{(-1)^{n}}{2 \pi i} \frac{\Gamma(n+N+2)}{\Gamma(n+1) \Gamma(N-n+1)} \\
& \times e^{N \gamma} \int_{-\infty}^{+\infty} \int_{+\infty}^{(0+)} \frac{h(u, \tau)}{u} e^{N \widetilde{\psi}(u)-N \tau^{2}} \mathrm{~d} u \mathrm{~d} \tau,
\end{aligned}
$$

where

$$
h(u, \tau)=\frac{u}{w-1} \frac{\mathrm{d} w}{\mathrm{~d} u} \frac{\mathrm{d} t}{\mathrm{~d} \tau} .
$$

From (3.27), we have

$$
\begin{aligned}
\frac{\mathrm{d} w}{\mathrm{~d} u} & =\frac{a-u}{u} / \frac{\mathrm{d} \tilde{f}\left(t_{0}(w), w\right)}{\mathrm{d} w} \\
& =\frac{(a-u) w(1-w)\left[(1-2 a)+\sqrt{1+4 b^{2} w^{2}-4 b^{2} w}\right]}{2 u b^{2}\left(w-w_{+}\right)\left(w-w_{-}\right)}
\end{aligned}
$$

for $u \neq a$, and by l'Hôpital's rule

$$
\frac{\mathrm{d} w}{\mathrm{~d} u}=\left\{\frac{(1-a)(1-2 a)}{b^{3} \sqrt{b^{2}-4 a+4 a^{2}}}\right\}^{1 / 2}
$$

for $u=a$ 


\section{THE MAPPING $w \rightarrow u$ IN (3.15)}

Before establishing the existence and uniqueness of the constants $\eta$ and $\gamma$ in equation (3.15) which defines the mapping $w \rightarrow u$, we first need a preliminary result.

Lemma 1. Let $0 \leq a \leq \frac{1}{2}$, and put $g(\eta):=\psi\left(u_{-}\right)-\psi\left(u_{+}\right)$when $a_{-}<a \leq \frac{1}{2}$, where $u_{ \pm}=\left(\eta \pm \sqrt{\eta^{2}+4 a \eta}\right) / 2 \eta$ are given in 3.14); put $g_{ \pm}(\eta):=\psi\left(u_{-}^{( \pm)}\right)-\psi\left(u_{+}^{( \pm)}\right)$when $0 \leq a \leq a_{-}$, where $u_{ \pm}^{(+)}$denote the $u_{ \pm}$on the upper edge of the real line and $u_{ \pm}^{(-)}$denote the $u_{ \pm}$on the lower edge of the real line; see Figure 7 . We have

(i) when $\eta \leq-4 a, g_{+}(\eta)=g_{-}(\eta)=k(\eta)$, where $k(\eta)$ is a real and monotonically increasing function with range $(-\infty, 0]$;

(ii) when $-4 a<\eta<0, g(\eta)$ is purely imaginary and -ig( $\eta)$ is a monotonically increasing and continuous function with range $(-2 a \pi, 0)$.

Proof. In case (i), we have $\eta^{2}+4 a \eta>0$. By definition,

$$
g_{ \pm}(\eta)=a \ln u_{-}^{( \pm)}-a \ln \left(u_{-}^{( \pm)}-1\right)+\eta u_{-}^{( \pm)}-a \ln u_{+}^{( \pm)}+a \ln \left(u_{+}^{( \pm)}-1\right)-\eta u_{+}^{( \pm)} .
$$

Recall the movements of $u_{ \pm}$shown in Figure 7. Since $u_{ \pm}^{(-)}$lie on the lower edge of the cut $[0,1]$, it follows that

$$
u_{ \pm}^{(-)}-1=\left|u_{ \pm}^{(-)}-1\right| e^{-\pi i}=\frac{\eta-\sqrt{\eta^{2}+4 a \eta}}{2 \eta} e^{-\pi i} .
$$

Similarly, since $u_{ \pm}^{(+)}$lie on the upper edge of the cut, we have

$$
u_{ \pm}^{(+)}-1=\left|u_{ \pm}^{(+)}-1\right| e^{\pi i}=\frac{\eta+\sqrt{\eta^{2}+4 a \eta}}{2 \eta} e^{\pi i} .
$$

Put

$$
k(\eta):=2 a \ln \frac{\eta-\sqrt{\eta^{2}+4 a \eta}}{\eta+\sqrt{\eta^{2}+4 a \eta}}-\sqrt{\eta^{2}+4 a \eta} .
$$

Then

$$
g_{+}(\eta)=g_{-}(\eta)=k(\eta)
$$

Let $\xi(\eta)=\sqrt{\eta^{2}+4 a \eta} / \eta$. It is easily checked that $\xi(\eta)$ is a monotonically increasing function in $-\infty<\eta \leq-4 a$ with range $(-1,0]$. If

$$
\widetilde{k}(\xi):=2 a \ln \frac{1-\xi}{1+\xi}+4 a \frac{\xi}{1-\xi^{2}}
$$

then,

$$
\widetilde{k}^{\prime}(\xi)=\frac{8 \xi^{2} a}{\left(1-\xi^{2}\right)^{2}}>0,
$$

i.e., $\widetilde{k}(\xi)$ is monotonically increasing in $-1<\xi \leq 0$. Since $k(\eta)=\widetilde{k}(\xi(\eta))$, we conclude that $k(\eta)$ is monotonically increasing in $-\infty<\eta \leq-4 a$ with range $(-\infty, 0]$. Note that $k(\eta)=0$ when $\eta=-4 a$, i.e., $a=a_{-}$. 
In case (ii), we have $\eta^{2}+4 a \eta<0$. Hence,

$$
u_{ \pm}=\frac{\eta \pm i \sqrt{-4 a \eta-\eta^{2}}}{2 \eta} .
$$

From (3.13), it is clear that $\operatorname{Re} \psi\left(u_{+}\right)=\operatorname{Re} \psi\left(u_{-}\right)$and $\operatorname{Im} \psi\left(u_{+}\right)=-\operatorname{Im} \psi\left(u_{-}\right)$. Thus, $g(\eta)$ is purely imaginary. Since $\eta$ is negative, we have

$$
\begin{aligned}
g(\eta) & =i 2 \operatorname{Im}\left[a \ln \frac{\eta-i \sqrt{-\eta^{2}-4 a \eta}}{2 \eta}-a \ln \frac{-\eta-i \sqrt{-\eta^{2}-4 a \eta}}{2 \eta}\right]-i \sqrt{-\eta^{2}-4 a \eta} \\
& =i\left[2 a\left(\arctan \frac{\sqrt{-\eta^{2}-4 a \eta}}{-\eta}-\pi+\arctan \frac{\sqrt{-\eta^{2}-4 a \eta}}{-\eta}\right)-\sqrt{-\eta^{2}-4 a \eta}\right] .
\end{aligned}
$$

Since $-i g^{\prime}(\eta)=-\sqrt{-\eta^{2}-4 a \eta} / \eta>0$, the function $-i g(\eta)$ is monotonically increasing. Furthermore, since $g(-4 a)=-2 \pi a i$ and $g(0)=0$, the range of this function is $(-2 a \pi, 0)$.

Theorem 1. Let $0 \leq a \leq \frac{1}{2}$, (i) When $a_{-}<a \leq \frac{1}{2}$, there exist unique real numbers $\eta$ and $\gamma$ satisfying the system of equations $u\left(w_{+}\right)=u_{-}$and $u\left(w_{-}\right)=u_{+}$in (3.16), i.e,

$$
\begin{gathered}
b \ln \left(1-t_{+}\right)+(1-b) \ln t_{+}+a \ln w_{+}-a \ln \left(w_{+}-1\right)+b \ln \left[1-\left(1-t_{+}\right) w_{+}\right] \\
=a \ln u_{-}-a \ln \left(u_{-}-1\right)+\eta u_{-}+\gamma \\
b \ln \left(1-t_{-}\right)+(1-b) \ln t_{-}+a \ln w_{-}-a \ln \left(w_{-}-1\right)+b \ln \left[1-\left(1-t_{-}\right) w_{-}\right] \\
=a \ln u_{+}-a \ln \left(u_{+}-1\right)+\eta u_{+}+\gamma ;
\end{gathered}
$$

see also (2.7) and (3.15). (ii) When $0 \leq a \leq a_{-}$, there exist unique real numbers $\eta$ and $\gamma$ satisfying the system of equations in (3.17a) (since (3.17b) is equivalent to (3.17a)), i.e,

$$
\begin{gathered}
b \ln \left(1-t_{+}\right)+(1-b) \ln t_{+}+a \ln w_{+}^{(+)}-a \ln \left(w_{+}^{(+)}-1\right)+b \ln \left[1-\left(1-t_{+}\right) w_{+}^{(+)}\right] \\
=a \ln u_{-}^{(+)}-a \ln \left(u_{-}^{(+)}-1\right)+\eta u_{-}^{(+)}+\gamma, \\
b \ln \left(1-t_{-}\right)+(1-b) \ln t_{-}+a \ln w_{-}^{(+)}-a \ln \left(w_{-}^{(+)}-1\right)+b \ln \left[1-\left(1-t_{-}\right) w_{-}^{(+)}\right] \\
=a \ln u_{+}^{(+)}-a \ln \left(u_{+}^{(+)}-1\right)+\eta u_{+}^{(+)}+\gamma ;
\end{gathered}
$$

Proof. When $0 \leq a \leq a_{-}$, we denote the left-hand sides and the right-hand sides of the two equations in (4.2) by $L_{1}, L_{2}$ and $R_{1}, R_{2}$, respectively. We recall that both $w_{ \pm}^{(+)}$are real and lie in the interval $[0,1]$, and that they move along the upper edge of the real line; see Figure 5. Thus, in $L_{1}$ and $L_{2}$, we have

$$
a \ln \left(w_{ \pm}^{(+)}-1\right)=a \ln \left(1-w_{ \pm}^{(+)}\right)+a \pi i .
$$

In this case, since $0<t_{ \pm}<1$ and $0<w_{ \pm}^{(+)}<1$, it can easily be verified that $1-(1-$ $\left.t_{ \pm}\right) w_{ \pm}^{(+)}>0$. Hence, the terms in $L_{1}$ and $L_{2}$ are well defined. Furthermore, since all terms containing $t_{ \pm}$are real, we have $L_{1}-L_{2}=\operatorname{Re} L_{1}-\operatorname{Re} L_{2}$. Also, for $0<w<1$,

$$
\begin{aligned}
\operatorname{Re} f\left(t_{0}(w), w\right)= & b \ln \left(1-t_{0}(w)\right)+(1-b) \ln t_{0}(w)+a \ln w \\
& -a \ln (1-w)+b \ln \left[1-\left(1-t_{0}(w)\right) w\right],
\end{aligned}
$$


which is a real-valued function. It can be shown that $\operatorname{Re} f\left(t_{0}(w), w\right)$ attains its maximum value at $w=w_{-}$. Hence, $\operatorname{Re} f\left(t_{-}, w_{-}\right)>\operatorname{Re} f\left(t_{+}, w_{+}\right)$. and there exists a non-positive number $r(a)$ such that $L_{1}-L_{2}=r(a)$. By Lemma 1)(i), $g_{+}(\eta)=k(\eta)$, where $k(\eta)$ is a real-valued monotonically increasing function with range $(-\infty, 0]$. Therefore, for each $a \in\left[0, a_{-}\right]$, there must exist a unique $\eta$ such that $k(\eta)=r(a)$ and the equations in (4.2) hold. The value of $\gamma$ is then determined by either one of the two equations in (4.2).

When $a_{-}<a \leq \frac{1}{2}$, we also denote the left-hand sides and right-hand sides of the two equations in (4.1) by $L_{1}, L_{2}$ and $R_{1}, R_{2}$, respectively. Recall that $w_{ \pm}$are complex conjugate numbers. Since $\overline{f(t, w)}=f(\bar{t}, \bar{w})$, from (4.1) we have $L_{1}-L_{2}=q(a) i$, where

$$
\begin{aligned}
q(a)= & 2 \operatorname{Im}\left\{b \ln \left(1-t_{+}\right)+(1-b) \ln t_{+}+a \ln w_{+}\right. \\
& \left.-a \ln \left(w_{+}-1\right)+b \ln \left[1-\left(1-t_{+}\right) w_{+}\right]\right\} .
\end{aligned}
$$

Straightforward calculation gives

$$
\begin{aligned}
q(a)=2[ & 2 b \arctan \frac{-\sqrt{4 a-4 a^{2}-b^{2}}}{2-2 a+b}+(1-b) \arctan \frac{b \sqrt{4 a-4 a^{2}-b^{2}}}{2-2 a-b^{2}} \\
& \left.+2 a \arctan \frac{\sqrt{4 a-4 a^{2}-b^{2}}}{b}-a \pi\right] .
\end{aligned}
$$

Note that in the present case, $4 a-4 a^{2}-b^{2} \geq 0$. Also, we have

$$
q^{\prime}(a)=-2 \pi+4 \arctan \frac{\sqrt{4 a-4 a^{2}-b^{2}}}{b}<0 .
$$

Thus, $q(a)<q\left(a_{-}\right)=-2 \pi a_{-}<0$. Now, consider the function $q(a)+2 \pi a$. Since

$$
\frac{\mathrm{d}}{\mathrm{d} a}(q(a)+2 \pi a)=4 \arctan \frac{\sqrt{4 a-4 a^{2}-b^{2}}}{b}>0,
$$

it follows that $q(a)+2 \pi a>q\left(a_{-}\right)+2 \pi a_{-}=0$ and hence $q(a)>-2 \pi a$. Coupling these two results, we obtain $-2 \pi a<q(a)<0$. From the right-hand sides of the two equations in (4.1), by Lemma 1(ii) we have $R_{1}-R_{2}=r(\eta) i$, where $r(\eta)$ is a real-valued increasing function with range $(-2 a \pi, 0)$. Therefore, for every fix $a \in\left(a_{-}, \frac{1}{2}\right]$, there exists a unique $\eta$ such that $r(\eta)=q(a)$ and the system of equations in (4.1) holds. The value of $\gamma$ is again determined by either one of the two equations in (4.1).

Theorem 2. The mapping $w \rightarrow u$ defined in (3.15) is one-to-one and analytic when $0 \leq a \leq \frac{1}{2}$.

Proof. As in the cases of Charlier polynomials [7] and Meixner polynomials [8], we introduce an intermediate variable $Z$ defined by

$$
\begin{gathered}
b \ln \left(1-t_{0}\right)+(1-b) \ln t_{0}+a \ln w-a \ln (w-1)+b \ln \left[1-\left(1-t_{0}\right) w\right] \\
=Z=a \ln u-a \ln (u-1)+\eta u+\gamma,
\end{gathered}
$$

where $t_{0}=t_{0}(w)$ is the relavant saddle point $t_{0}^{+}(w)$ given in (3.4). To establish the properties of the mapping $w \rightarrow u$, we again divide our discussion into two cases: $0 \leq a<a_{-}$ 


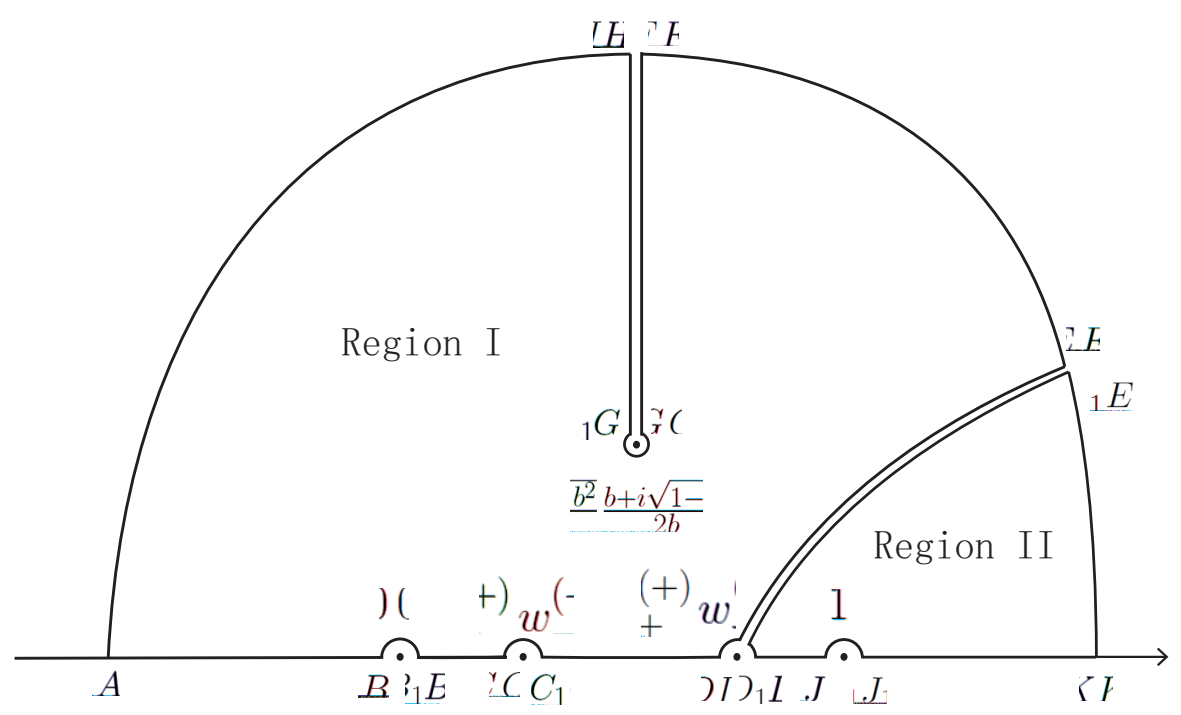

Figure 9: The upper half of the $w$-plane.

and $a_{-}<a \leq \frac{1}{2}$

When $0 \leq a<a_{-}$, we consider the upper half of the $w$-plane. Since the functions $f\left(t_{0}(w), w\right)$ and $\psi(u)+\gamma$ are both symmetric with respect to the real line, the case for the lower half of the $w$-plane can be handled in the same manner. To avoid the multivaluedness around the saddle point, we divide the upper half of the $w$-plane into two parts by using the steepest path through $w_{+}^{(+)}$; see Figure 9, Call these parts region I and region II. Note that there are branch cuts, one along the infinite interval $(-\infty, 1]$ and the other along the vertical line Rew $=\frac{1}{2}$ from $\left(b+i \sqrt{1-b^{2}}\right) / 2 b$ to $\frac{1}{2}+i \infty$. The cut along the vertical line was introduced in (3.4).

As $w$ traverses along the boundary $A B B_{1} C C_{1} D D_{1} E F G G_{1} H A$ of region I, the image point $Z$ traverses along the corresponding boundary of a region in the $Z$-plane; see Figure 10. In Figure 12, we draw the boundary of the region, corresponding to region I, in the $u$-plane. The image point $Z=\psi(u)+\gamma$ traverses along the boundary of the same region shown in Figure 10.

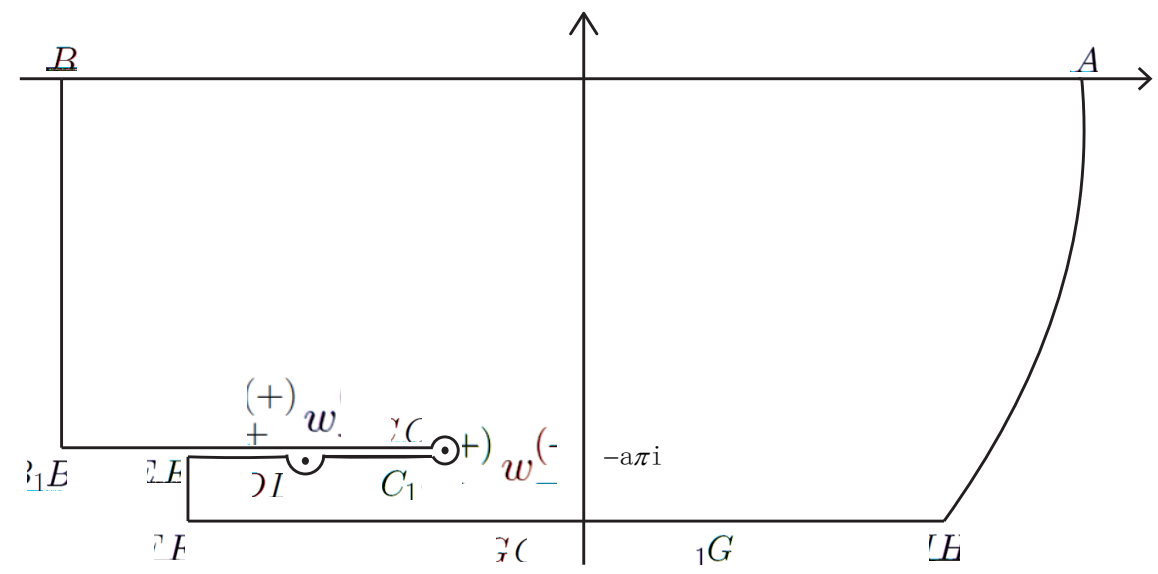

Figure 10: The image of Region I in the $Z$-plane. 


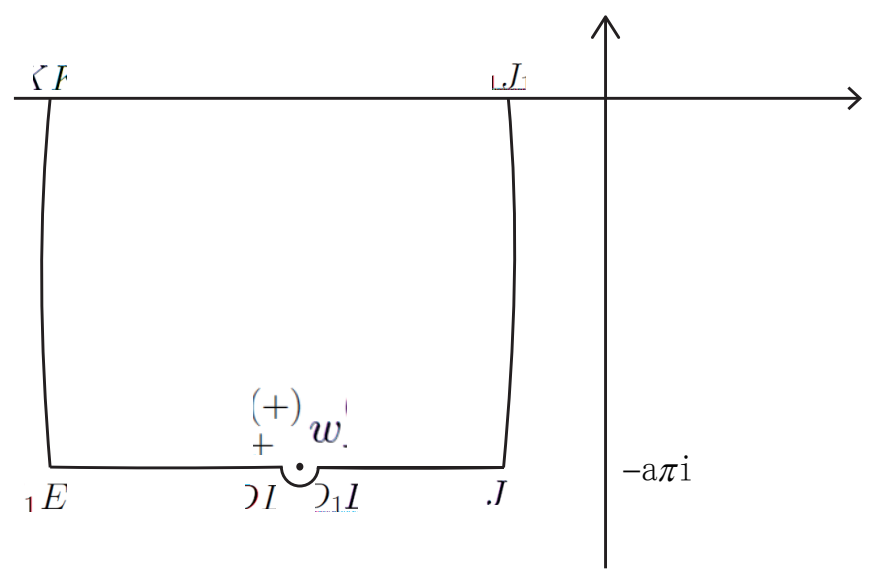

Figure 11: The image of Region II in the $Z$-plane.

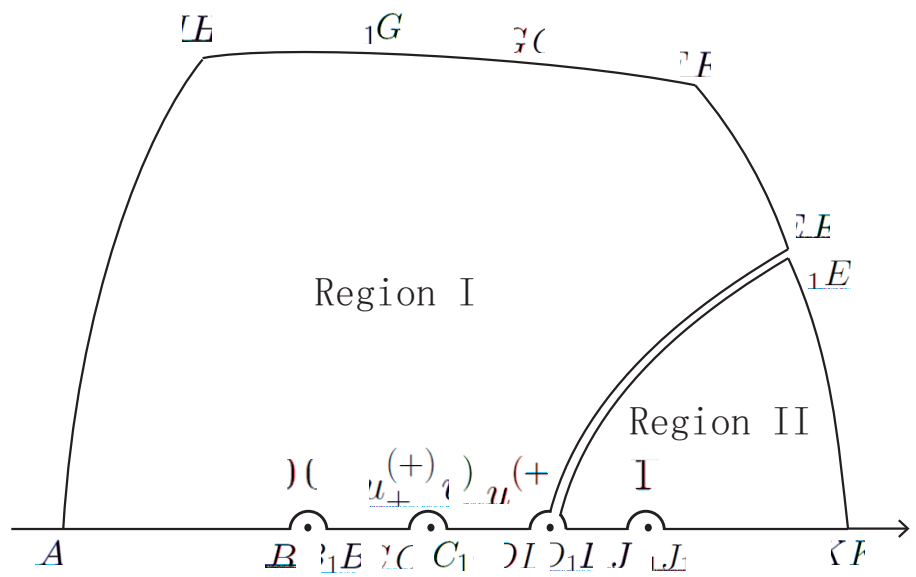

Figure 12: The upper half of the $u$-plane.

Let $\varphi(w)=f\left(t_{0}(w), w\right)$ so that (4.3) becomes $\varphi(w)=\psi(u)+\gamma$. Since the mapping $w \rightarrow u$ is just the composite function $\varphi^{-1}$ and $\psi(u)+\gamma$, it is one-to-one on the boundary of region I. By the same argument, one can prove that this mapping is also one-to-one on the boundary of region II. For the image of region II in the intermediate $Z$-plane, see Figure 11, By Theorem 1.2.2 of [20, p.12], the mapping $w \rightarrow u$ is one-to-one in the interior of both regions. As explained earlier, the one-to-one property of this mapping in the lower half of the $w$-plane can be established by using symmetry of the functions with respect to the real axis. Note that the only possible singular points of the mapping $w \rightarrow u$ are at $w=0,1$ and $w_{ \pm}^{(+)}$. Since the images of these points in the $u$-plane are bounded (see the points 0,1 and $u_{ \pm}^{(+)}$in Figure 12), the mapping is indeed one-to-one and analytic in the whole $w$-plane.

When $a_{-}<a \leq \frac{1}{2}$, the one-to-one and analytic properties of the mapping $w \rightarrow u$ can be proved in the same manner as in the previous case. Hence, we simply skip this part of the argument.

Thus far, we have proved the one-to-one and analytic nature of the mapping $w \rightarrow u$ defined in (3.15) when $0 \leq a<a_{-}$and when $a_{-}<a \leq \frac{1}{2}$. To show that it also has these properties at $a=a_{-}$, we note that equation (3.15) can be written as $g(u, \eta, \gamma, f)=0$, 
where $g$ is just the difference between the left-hand side and the right-hand side of the equation. Since $\eta, \gamma$ and $f\left(t_{0}(w), w\right)$ are all continuous in $a$ (see the proof of Theorem 1 ), by the implicit function theorem $u$ is continuous in $a$ except at $u_{ \pm}$, where $\partial g / \partial u$ vanishes. The continuity of $u$ in $a$ when $u=u_{ \pm}$comes from (3.14) and the continuity of $\eta$. Therefore, the mapping is also one-to-one and analytic when $a=a_{-}$.

\section{ANALYTICITY OF $h(u, \tau)$}

Before deriving the asymptotic expansions of the integrals in (3.20) and (3.29), we first investigate the analyticity of the amplitude function $h(u, \tau)$ in the neighborhood of the steepest descent surface $S_{\tau \times u}=(-\infty, \infty) \times \gamma_{u}$; cf. the statements following (3.22). For an expression of this function, see (3.21) for the case $0 \leq a \leq \frac{1}{2}$, and (3.30) for the case $a<0$.

Case 1: $0 \leq a \leq \frac{1}{2}$

For $w \neq w_{ \pm}$and $t \neq t_{0}^{+}(w)$ (i.e., $\tau \neq 0$ ), we have from (3.21), (3.18) and (3.7)

$$
\begin{aligned}
h(u, \tau)= & \frac{u-1}{w-1} \frac{2 \tau t(1-t)[1-(1-t) w]}{(1+b) w\left(t-t_{0}^{+}(w)\right)\left(t-t_{0}^{-}(w)\right)} \\
& \times \frac{\eta\left(u-u_{+}\right)\left(u-u_{-}\right) w(1-w)\left[(2 a-1)-\sqrt{1+4 b^{2} w^{2}-4 b^{2} w}\right]}{-2 b^{2} u(u-1)\left(w-w_{+}\right)\left(w-w_{-}\right)} .
\end{aligned}
$$

Since $\rho_{t} \times \gamma_{w}$ in the $t \times w$-plane does not pass through $t_{0}^{-}(w)$ (unless $t_{0}^{-}(w)$ coalesces with $t_{0}^{+}(w)$, a case which we will discuss next), we may substitute (3.4) in (5.1) to give

$$
\begin{aligned}
h(u, \tau)= & \frac{2 w \tau t(1-t)[1-(1-t) w]}{\left[2(1+b) w t-2 w+1+\sqrt{1+4 b^{2}(w-1) w}\right]\left(t-t_{0}^{+}(w)\right)} \\
& \times \frac{\eta\left(u-u_{+}\right)\left(u-u_{-}\right) w\left[(2 a-1)-\sqrt{1+4 b^{2} w^{2}-4 b^{2} w}\right]}{b^{2} u\left(w-w_{+}\right)\left(w-w_{-}\right)} .
\end{aligned}
$$

Thus, in the neighborhood of the steepest descent surface $S_{\tau \times u}, h(\tau, u)$ is analytic except possibly at points $u=0$ and $u=1$, which correspond to $w=0$ and $w=1$, respectively. From (3.4), it is easily verified that $t_{0}^{+}(w) \rightarrow 1-b$ when $w \rightarrow 0$ and $t_{0}^{+}(w) \rightarrow \frac{1}{1+b}$ when $w \rightarrow 1$. Substituting these values into (3.15) or (3.22) yields $\frac{w}{u}=O(1)$ as $w \rightarrow 0$ and $\frac{w-1}{u-1}=O(1)$ as $w \rightarrow 1$. Hence, neither $u=0$ nor $u=1$ is the singular point in this case.

If $w=w_{ \pm}$but $t \neq t_{0}^{+}(w)$, then in (3.21) we use (3.19) instead of (3.18) to arrive at

$$
\begin{aligned}
h(u, \tau)= & \frac{u_{\mp}-1}{w_{ \pm}-1} \frac{2 \tau t(1-t)\left[1-(1-t) w_{ \pm}\right]}{(1+b) w_{ \pm}\left(t-t_{ \pm}\right)\left(t-t_{0}^{-}\left(w_{ \pm}\right)\right)} \\
& \times\left\{\frac{\sqrt{\eta^{2}+4 a \eta}(1-a)(1-2 a)(-\eta)}{b^{3} \sqrt{b^{2}-4 a+4 a^{2}}}\right\}^{1 / 2} .
\end{aligned}
$$

Since we are in the case $0 \leq a \leq \frac{1}{2}$, we have $w_{-} \neq 1$ (i.e., $u_{+} \neq 1$ ), $w_{+} \neq 0$ (i.e., $u_{-} \neq 0$ ) and $a \neq \frac{1}{2}\left(1+\sqrt{1-b^{2}}\right)$. Thus, $u_{+}=0, u_{-}=1$, and $a=\frac{1-\sqrt{1-b^{2}}}{2}$ are the only possible singularities, which by (3.15) and (3.16) correspond to $w_{-}=0, w_{+}=1$ and $\eta=-4 a$, 
respectively. It can be also verified that $\frac{w_{-}}{u_{+}}=O(1)$ as $u_{+} \rightarrow 0, \frac{w_{+}-1}{u_{-}-1}=O(1)$ as $u_{-} \rightarrow 1$, and $\sqrt{\eta^{2}+4 a \eta} / \sqrt{b^{2}-4 a+4 a^{2}}=O(1)$ as $a \rightarrow \frac{1}{2}\left(1-\sqrt{1-b^{2}}\right)$. Hence, these possible singularities are actually removable.

If $w \neq w_{ \pm}$but $t=t_{0}^{+}(w)$, then in (3.21) we use the second equation (instead of the first one) in (3.7) to reach

$$
\begin{aligned}
h(u, \tau)= & \left\{\frac{2 t_{0}^{+}(w)\left(1-t_{0}^{+}(w)\right)\left[1-\left(1-t_{0}^{+}(w)\right) w\right]}{\sqrt{1+4 b^{2}(w-1) w}}\right\}^{1 / 2} \\
& \times \frac{\eta\left(u-u_{+}\right)\left(u-u_{-}\right) w\left[(2 a-1)-\sqrt{1+4 b^{2} w^{2}-4 b^{2} w}\right.}{2 b^{2} u\left(w-w_{+}\right)\left(w-w_{-}\right)},
\end{aligned}
$$

where, in addition to the possible singular point at $u=0$ which we have just discussed, we have introduced two new possible singularities $w=\left(b \pm i \sqrt{1-b^{2}}\right) / 2 b$. However, from Figure 1 it is clear that these two points will not be in the neighborhood of $\rho_{t} \times \gamma_{w}$, unless $a \rightarrow \frac{1}{2}$ in which case $w \rightarrow w_{ \pm}$(see the discussion below).

If $w=w_{ \pm}$and $t=t_{0}^{+}(w)$, then in (3.21) we use (3.19) and the second equation in (3.7). Since $t_{0}^{+}\left(w_{ \pm}\right)=t_{ \pm}$, the result is

$$
\begin{aligned}
h(u, \tau)= & \frac{u_{\mp}-1}{w_{ \pm}-1}\left\{\frac{2 t_{ \pm}\left(1-t_{ \pm}\right)\left[1-\left(1-t_{ \pm}\right) w_{ \pm}\right]}{b^{3}}\right. \\
& \left.\times \frac{\sqrt{\eta^{2}+4 a \eta}(1-a)(1-2 a)(-\eta)}{\sqrt{b^{2}-4 a+4 a^{2}}}\right\}^{1 / 2} .
\end{aligned}
$$

Note that $a=\frac{1}{2}$ is not a singular point, and since $\sqrt{\eta^{2}+4 a \eta} / \sqrt{b^{2}-4 a+4 a^{2}}=O(1)$ as $a \rightarrow \frac{1}{2}\left(1-\sqrt{1-b^{2}}\right)$ as we have discussed before, there is no singular point in the neighborhood of the integration surface.

Case 2: $a<0$.

In this case, only the negative saddle point $w_{-}$is relevant, and the steepest descent path associated with $w_{-}$will not pass through $w_{+}$. Thus, the situation is much simpler than the case $0 \leq a \leq \frac{1}{2}$. By using (3.30), (3.7), (3.31) and (3.32), we have the following analytic expressions for $h(u, \tau)$ :

If $w \neq w_{-}$and $t \neq t_{0}^{+}(w)$, we have

$$
h(u, \tau)=\frac{(u-a)\left[(1-2 a)+\sqrt{1+4 b^{2} w^{2}-4 b^{2} w}\right]}{b^{2}\left(w-w_{+}\right)\left(w-w_{-}\right)} \frac{\tau t(1-t)[1-(1-t) w]}{(1+b)\left(t-t_{0}^{+}(w)\right)\left(t-t_{0}^{-}(w)\right)} .
$$

Since $w \neq w_{-}$and $t \neq t_{0}^{+}(w)$, clearly there is no singularity in the neighborhood of the integration surface.

If $w \neq w_{-}$but $t=t_{0}^{+}(w)$, then

$h(u, \tau)=\left\{\frac{2 t_{0}^{+}(w)\left(1-t_{0}^{+}(w)\right)\left[1-\left(1-t_{0}^{+}(w)\right) w\right]}{\sqrt{1+4 b^{2}(w-1) w}}\right\}^{1 / 2} \frac{(u-a) w\left[(1-2 a)+\sqrt{1+4 b^{2} w^{2}-4 b^{2} w}\right]}{2 b^{2}\left(w-w_{+}\right)\left(w-w_{-}\right)}$. 
Again, there is no singularity.

If $w=w_{-}$(in which case $u=a$ by (3.28) $)$ but $t \neq t_{0}^{+}(w)$, we have

$$
h(u, \tau)=\frac{-2 \tau t(1-t)\left[1-(1-t) w_{-}\right]}{(1+b)\left(t-t_{-}\right)\left(t-t_{0}^{-}\left(w_{-}\right)\right)}\left\{\frac{a b(1-2 a)}{(1-a) \sqrt{b^{2}-4 a-4 a^{2}}}\right\}^{1 / 2} .
$$

The only possible singularity occurs when $w_{-}=0$ (i.e., when $a \rightarrow 0^{-}$). Since it can be shown that $\frac{a}{w_{-}}=O(1)$ as $a \rightarrow 0^{-}, h(u, \tau)$ is again bounded and analytic as $a \rightarrow 0^{-}$.

If $w=w_{-}$and $t=t_{0}^{+}(w)$, then

$$
h(u, \tau)=\left\{\frac{2 a w_{-} t_{-}\left(1-t_{-}\right)\left[1-\left(1-t_{-}\right) w_{-}\right]}{\left(1-w_{-}\right) b \sqrt{b^{2}-4 a-4 a^{2}}}\right\}^{1 / 2}
$$

and there is no possible singularity at all.

The conclusions in all these cases infer that for $a<0, h(u, \tau)$ is analytic in the neighborhood of the surface of integration.

\section{ASYMPTOTIC EXPANSIONS}

Case 1: $0 \leq a \leq \frac{1}{2}$

Let $h_{0}(u, \tau) \equiv h(u, \tau)$, where $h(u, \tau)$ is the amplitude function given in (3.21). Write

$$
h_{0}(u, \tau)=a_{0}(\tau)+b_{0}(\tau) u-\left(u-u_{+}\right)\left(u-u_{-}\right) g_{0}(u, \tau)
$$

Clearly,

$$
\begin{aligned}
& a_{0}(\tau)=\frac{u_{-} h_{0}\left(u_{+}, \tau\right)-u_{+} h_{0}\left(u_{-}, \tau\right)}{u_{-}-u_{+}}, \\
& b_{0}(\tau)=\frac{h_{0}\left(u_{-}, \tau\right)-h_{0}\left(u_{+}, \tau\right)}{u_{-}-u_{+}}
\end{aligned}
$$

and

$$
g_{0}(u, \tau)=\frac{1}{\left(u-u_{+}\right)\left(u-u_{-}\right)}\left[h_{0}(u, \tau)-a_{0}(\tau)-b_{0}(\tau) u\right]
$$

For $u \neq u_{ \pm}, g_{0}(u, \tau)$ is analytic in $u$ wherever $h_{0}(u, \tau)$ is analytic. Furthermore, it is easily shown that the limits

$$
\begin{aligned}
& \lim _{u \rightarrow u_{+}} g_{0}(u, \tau)=\frac{h_{0}^{\prime}\left(u_{+}, \tau\right)}{u_{+}-u_{-}}-\frac{h_{0}\left(u_{+}, \tau\right)-h_{0}\left(u_{-}, \tau\right)}{\left(u_{+}-u_{-}\right)^{2}} \quad \text { if } \quad a \neq a_{-}, \\
& \lim _{u \rightarrow u_{-}} g_{0}(u, \tau)=\frac{h_{0}^{\prime}\left(u_{-}, \tau\right)}{u_{-}-u_{+}}+\frac{h_{0}\left(u_{+}, \tau\right)-h_{0}\left(u_{-}, \tau\right)}{\left(u_{+}-u_{-}\right)^{2}} \quad \text { if } \quad a \neq a_{-}, \\
& \lim _{u \rightarrow u_{+}} g_{0}(u, \tau)=\lim _{u \rightarrow u_{-}} g_{0}(u, \tau)=\frac{1}{2} h_{0}^{\prime \prime}\left(u_{-}, \tau\right) \quad \text { if } \quad a=a_{-},
\end{aligned}
$$


exist and are finite. Thus, $g_{0}(u, \tau)$ is actually analytic in $u$ everywhere $h_{0}(u, \tau)$ is analytic.

Recall the integral representation of the confluent hypergeometric function $\mathbf{M}(a N+$ $1,1, \eta N)$ in (3.12)

$$
\mathbf{M}(a N+1,1, \eta N)=\frac{1}{2 \pi i} \int_{\gamma_{1}} e^{N \psi(u)} \frac{\mathrm{d} u}{u-1},
$$

where $\psi(u)=a \ln u-a \ln (u-1)+\eta u$ and $\gamma_{1}$ is the loop contour which starts at $u=0$, encircles $u=1$ in the positive direction, and returns to $u=0$; see (2.5). From (3.11), we also have

$$
\mathbf{M}^{\prime}(a N+1,1, \eta N)=\frac{1}{2 \pi i} \int_{\gamma_{1}} \frac{u}{u-1} e^{N \psi(u)} \mathrm{d} u
$$

where the derivative is taken with respect to the third variable in the function $\mathbf{M}(c, d, z)$. Inserting (6.1) in (3.20), we obtain

$$
\begin{aligned}
t_{n}(x, N+1)=\frac{(-1)^{n} \Gamma(n+N+2)}{\Gamma(n+1) \Gamma(N-n+1)} e^{N \gamma} & {\left[\mathbf{M}(a N+1,1, \eta N) \int_{-\infty}^{+\infty} a_{0}(\tau) e^{-N \tau^{2}} \mathrm{~d} \tau\right.} \\
& \left.+\mathbf{M}^{\prime}(a N+1,1, \eta N) \int_{-\infty}^{+\infty} b_{0}(\tau) e^{-N \tau^{2}} \mathrm{~d} \tau+\epsilon_{1}^{+}\right],
\end{aligned}
$$

where

$$
\epsilon_{1}^{+}=-\frac{1}{2 \pi i} \int_{-\infty}^{+\infty}\left[\int_{\gamma_{u}}\left(u-u_{+}\right)\left(u-u_{-}\right) g_{0}(u, \tau) e^{N \psi(u)} \frac{\mathrm{d} u}{u-1}\right] e^{-N \tau^{2}} \mathrm{~d} \tau .
$$

To the inner integral, we apply an integration-by-parts; the result is that it is equal to

$$
\left.\frac{1}{\eta N} u e^{N \psi(u)} g_{0}(u, \tau)\right|_{u=0^{-}} ^{u=0^{+}}-\frac{1}{\eta N} \int_{\gamma_{u}} h_{1}(u, \tau) e^{N \psi(u)} \frac{\mathrm{d} u}{u-1},
$$

where

$$
h_{1}(u, \tau)=(u-1)\left[g_{0}(u, \tau)+u \frac{\partial g_{0}(u, \tau)}{\partial u}\right] .
$$

By using (6.3) and the fact that $h_{0}(u)=O(1)$ as $u \rightarrow 0$ as we have shown in \$5, the first term above is actually zero. Thus,

$$
\epsilon_{1}^{+}=\frac{1}{2 \pi i \eta N} \int_{-\infty}^{+\infty} \int_{\gamma_{u}} h_{1}(u, \tau) \frac{e^{N \psi(u)}}{u-1} e^{-N \tau^{2}} \mathrm{~d} u \mathrm{~d} \tau
$$

The two integrals in (6.6) can be asymptotically evaluated by using Watson's lemma [21, p.20]. So, let us expand $a_{0}(\tau)$ and $b_{0}(\tau)$ into Maclaurin expansions

$$
a_{0}(\tau)=\sum_{j=0}^{\infty} a_{0, j} \tau^{j}, \quad b_{0}(\tau)=\sum_{j=0}^{\infty} b_{0, j} \tau^{j}
$$


Since

$$
\int_{-\infty}^{+\infty} \tau^{2 j} e^{-N \tau^{2}} \mathrm{~d} \tau=\frac{\Gamma\left(j+\frac{1}{2}\right)}{N^{-j-\frac{1}{2}}}, \quad \int_{-\infty}^{+\infty} \tau^{2 j+1} e^{-N \tau^{2}} \mathrm{~d} \tau=0
$$

we obtain

$$
\begin{aligned}
t_{n}(x, N+1)=\frac{(-1)^{n} \Gamma(n+N+2)}{\Gamma(n+1) \Gamma(N-n+1)} e^{N \gamma}\left\{\mathbf{M}(a N+1,1, \eta N)\left[\frac{c_{0}}{\sqrt{N}}+\delta_{1}^{+}\right]\right. \\
\left.+\mathbf{M}^{\prime}(a N+1,1, \eta N)\left[\frac{d_{0}}{\sqrt{N}}+\widetilde{\delta}_{1}^{+}\right]+\epsilon_{1}^{+}\right\},
\end{aligned}
$$

where $\delta_{1}^{+}$and $\widetilde{\delta}_{1}^{+}$denote error terms that are $O\left(N^{-3 / 2}\right)$.

The above procedure can be repeated. For $l=0,1,2, \cdots$, we define recursively

$$
\begin{aligned}
h_{l}(u, \tau) & =a_{l}(\tau)+b_{l}(\tau) u-\left(u-u_{-}\right)\left(u-u_{+}\right) g_{l}(u, \tau), \\
h_{l+1}(u, \tau) & =(u-1)\left[g_{l}(u, \tau)+u \frac{\partial g_{l}(u, \tau)}{\partial u}\right],
\end{aligned}
$$

and

$$
a_{l}(\tau)=\sum_{j=0}^{\infty} a_{l, j} \tau^{j}, \quad b_{l}(\tau)=\sum_{j=0}^{\infty} b_{l, j} \tau^{j}
$$

Using induction, one can show that $h_{l}(u, \tau)$ and $g_{l}(u, \tau)$ are both analytic and bounded as $u \rightarrow 0$. Hence,

$$
\begin{aligned}
t_{n}(x, N+1)=\frac{(-1)^{n} \Gamma(n+N+2) e^{N \gamma}}{\Gamma(n+1) \Gamma(N-n+1)}\left\{\mathbf{M}(a N+1,1, \eta N)\left[\sum_{l=0}^{p-1} \frac{c_{l}}{N^{l+\frac{1}{2}}}+\delta_{p}^{+}\right]\right. \\
\left.+\mathbf{M}^{\prime}(a N+1,1, \eta N)\left[\sum_{l=0}^{p-1} \frac{d_{l}}{N^{l+\frac{1}{2}}}+\widetilde{\delta}_{p}^{+}\right]+\epsilon_{p}^{+}\right\},
\end{aligned}
$$

where

$$
\begin{aligned}
c_{l} & =\sum_{m=0}^{l} a_{l-m, 2 m} \Gamma\left(\frac{2 m+1}{2}\right), \\
d_{l} & =\sum_{m=0}^{l} b_{l-m, 2 m} \Gamma\left(\frac{2 m+1}{2}\right), \\
\epsilon_{p}^{+} & =\frac{1}{2 \pi i \eta N} \frac{1}{N^{p}} \int_{-\infty}^{+\infty} \int_{\gamma_{u}} h_{p}(u, \tau) \frac{e^{N \psi(u)}}{u-1} e^{-N \tau^{2}} \mathrm{~d} u \mathrm{~d} \tau,
\end{aligned}
$$

and $\delta_{p}^{+}, \widetilde{\delta}_{p}^{+}$are error terms of order $O\left(N^{-p-1 / 2}\right)$. 
To prove that for fixed $b=n / N \in(0,1)$, (6.12) is a uniform asymptotic expansion in $a \in\left[0, \frac{1}{2}\right]$, we need to show that there are positive constants $A_{p}$ and $B_{p}$, independent of $a$, such that

$$
\left|\epsilon_{p}^{+}\right| \leq \frac{A_{p}}{N^{p+\frac{1}{2}}}|\mathbf{M}(a N+1,1, \eta N)|+\frac{B_{p}}{N^{p+\frac{1}{2}}}\left|\mathbf{M}^{\prime}(a N+1,1, \eta N)\right|
$$

Case 2: $a<0$.

Proceeding in the same manner as in Case 1 , we put $h_{0}(u, \tau) \equiv h(u, \tau)$, where $h(u, \tau)$ is given by (3.30), and write

$$
h_{0}(u, \tau)=a_{0}(\tau)+(u-a) g_{0}(u, \tau) .
$$

Since $\lim _{u \rightarrow a} g_{0}(u, \tau)=h_{0}^{\prime}(a, \tau), g_{0}(u, \tau)$ is analytic in $u$ wherever $h_{0}(u, \tau)$ is. By (3.29) and (3.25), we have

$$
t_{n}(x, N+1)=\frac{(-1)^{n} \Gamma(n+N+2) N^{-a N} e^{N \gamma}}{\Gamma(n+1) \Gamma(N-n+1) \Gamma(-a N+1)}\left[\int_{-\infty}^{+\infty} a_{0}(\tau) e^{-N \tau^{2}} \mathrm{~d} \tau+\epsilon_{1}^{-}\right]
$$

where

$$
\epsilon_{1}^{-}=\frac{N^{a N} \Gamma(-a N+1)}{2 \pi i} \int_{-\infty}^{+\infty}\left[\int_{+\infty}^{(0+)}(u-a) g_{0}(u, \tau) e^{N \widetilde{\psi}(u)} \frac{\mathrm{d} u}{u}\right] e^{-N \tau^{2}} \mathrm{~d} \tau
$$

and $\widetilde{\psi}(u)$ is given in (3.26). To the inner integral, we apply an integration-by-parts. The result is that it is equal to

$$
-\left.\frac{1}{N} g_{0}(u, \tau) e^{N \widetilde{\psi}(u)}\right|_{u=\infty} ^{u=\infty e^{2 \pi i}}+\frac{1}{N} \int_{+\infty}^{\left(0^{+}\right)} h_{1}(u, \tau) e^{N \widetilde{\psi}(u)} \frac{\mathrm{d} u}{u},
$$

where

$$
h_{1}(u, \tau)=u \frac{\partial g_{0}(u, \tau)}{\partial u} .
$$

Since $\widetilde{\psi}(u) \sim-u$ as $u \rightarrow+\infty$, the first term vanishes and we have

$$
\epsilon_{1}^{-}=\frac{N^{a N} \Gamma(-a N+1)}{2 \pi i N} \int_{-\infty}^{+\infty}\left[\int_{+\infty}^{\left(0^{+}\right)} h_{1}(u, \tau) e^{N \widetilde{\psi}(u)} \frac{\mathrm{d} u}{u}\right] e^{-N \tau^{2}} \mathrm{~d} \tau .
$$

The Laplace integral in (6.18) can again be asymptotically evaluated by using Watson's lemma, and the result is

$$
t_{n}(x, N+1)=\frac{(-1)^{n} \Gamma(n+N+2) N^{-a N} e^{N \gamma}}{\Gamma(n+1) \Gamma(N-n+1) \Gamma(-a N+1)}\left[\frac{a_{0,0}}{\sqrt{N}} \Gamma\left(\frac{1}{2}\right)+\epsilon_{1}^{-}+\delta_{1}^{-}\right],
$$

where $a_{0,0}$ is the constant term in the expansion of $a_{0}(\tau)$ and $\delta_{1}^{-}$denotes the error term resulting from Watson's lemma; see (6.8). 
The process can be repeated. For $l=0,1,2, \cdots$, we define

$$
\begin{aligned}
h_{l}(u, \tau) & =a_{l}(\tau)+(u-a) g_{l}(u, \tau), \\
h_{l+1}(u, \tau) & =u \frac{\partial g_{l}(u, \tau)}{\partial u}, \\
a_{l}(\tau) & =\sum_{j=0}^{+\infty} a_{l, j} \tau^{j} .
\end{aligned}
$$

As before, we arrive at

$$
t_{n}(x, N+1)=\frac{(-1)^{n} \Gamma(n+N+2) N^{-a N} e^{N \gamma}}{\Gamma(n+1) \Gamma(N-n+1) \Gamma(-a N+1)}\left[\sum_{l=0}^{p-1} \frac{c_{l}}{N^{l+\frac{1}{2}}}+\epsilon_{p}^{-}+\delta_{p}^{-}\right],
$$

where

$$
c_{l}=\sum_{m=0}^{l} a_{l-m, 2 m} \Gamma\left(\frac{2 m+1}{2}\right)
$$

and

$$
\varepsilon_{p}^{-}=\frac{N^{a N} \Gamma(-a N+1)}{2 \pi i N^{p}} \int_{-\infty}^{+\infty}\left[\int_{+\infty}^{\left(0^{+}\right)} h_{p}(u, \tau) e^{N \widetilde{\psi}(u)} \frac{\mathrm{d} u}{u}\right] e^{-N \tau^{2}} \mathrm{~d} \tau .
$$

The error term $\delta_{p}^{-}$comes from applying Watson's lemma to the functions $a_{l}(\tau), 0 \leq l \leq$ $p-1$, and is of order $O\left(N^{-p-\frac{1}{2}}\right)$. Thus, to prove that expansion (6.21) is uniform with respect to $a$, we only need to show that there exists a constant $M_{p}$, independent of $a$, such that

$$
\left|\epsilon_{p}^{-}\right| \leq \frac{M_{p}}{N^{p+\frac{1}{2}}}
$$

\section{ERROR ESTIMATES}

To estimate the error terms $\epsilon_{p}^{+}$and $\epsilon_{p}^{-}$, we shall actually provide two slightly stronger versions of the results stated in (6.16) and (6.24). Indeed, we shall show that the constants $A_{p}$ and $B_{p}$ in (6.16) are independent of $b \in[\delta, 1-\delta]$ and $a \in\left[0, \frac{1}{2}\right]$ for any $\delta>0$, and similarly that the constant $M_{p}$ in (6.24) is independent of $b \in[\delta, 1-\delta]$ and $a \in(-\infty, 0)$. That is, the expansion in (6.12) is uniform in $b \in[\delta, 1-\delta]$ and $a \in\left[0, \frac{1}{2}\right]$, and the expansion in (6.21) is uniform in $b \in[\delta, 1-\delta]$ and $a \in(-\infty, 0)$.

To proceed, we will divide our discussion into five cases:

(i) $a_{-}<a \leq \frac{1}{2}$ and $N^{\frac{2}{3}}(\eta+4 a)$ is unbounded;

(ii) $N^{\frac{2}{3}}(\eta+4 a)$ is bounded;

(iii) $0 \leq a<a_{-}, N^{\frac{2}{3}}(\eta+4 a)$ is unbounded and $a N$ is unbounded;

(iv) $a>0$ and $a N$ is bounded; 
(v) $a<0$.

From (3.15), we note that $a=a_{-}$corresponds $\eta=-4 a$.

Case (i) First we recall the following result of Fedoryuk [17, p.124-125]; consider the integral

$$
I(\lambda)=\int_{\gamma} f(z) e^{\lambda S(z)} \mathrm{d} z, \quad z=\left(z_{1}, \cdots, z_{n}\right) \in \mathbb{C}^{n},
$$

where $\lambda>0, \mathrm{~d} z=\mathrm{d} z_{1} \cdots \mathrm{d} z_{n}$ and $\gamma$ is a smooth manifold of (real) dimension $n$. Assume that the function $f(z)$ and $S(z)$ are analytic in some domain $D$ containing $\gamma$. A point $z^{0}$ is called a saddle point of $S(z)$ if $\nabla S\left(z^{0}\right)=0$, and a simple saddle point if $\operatorname{det} S^{\prime \prime}\left(z^{0}\right) \neq 0$.

Theorem 3. Let $\max _{z \in \gamma} \operatorname{Re} S(z)$ be attained only at a point $z^{0}$, which is a simple saddle point and an interior point of $\gamma$. Then, as $\lambda \rightarrow+\infty$, we have

$$
I(\lambda)=\left(\frac{2 \pi}{\lambda}\right)^{n / 2}\left[\operatorname{det} S^{\prime \prime}\left(z^{0}\right)\right]^{-1 / 2} e^{\lambda S\left(z^{0}\right)}\left[f\left(z^{0}\right)+O\left(\frac{1}{\lambda}\right)\right] .
$$

The choice of the branch for the root depends on the orientation of the integration surface.

In our present case, $n=2$ and there are two simple saddle points $\left(u_{+}, 0\right)$ and $\left(u_{-}, 0\right)$. By Theorem 3, it follows from (6.15) that as $N \rightarrow \infty$

$$
\begin{aligned}
\epsilon_{p}^{+} \sim-\frac{(\eta+4 a)^{-1 / 4} e^{N \eta / 2}}{\sqrt{2} i(-\eta)^{5 / 4} N^{p+2}}[ & h_{p}\left(u_{+}, 0\right) e^{i\left(a N \pi-2 a N \varphi_{-}+\frac{1}{2} N \sqrt{-\eta^{2}-4 a \eta}+\frac{3 \pi}{4}-\varphi_{-}\right)} \\
& \left.+h_{p}\left(u_{-}, 0\right) e^{-i\left(a N \pi-2 a N \varphi_{-}+\frac{1}{2} N \sqrt{-\eta^{2}-4 a \eta}+\frac{3 \pi}{4}-\varphi_{-}\right)}\right]
\end{aligned}
$$

where $\varphi_{-}$is the argument of $u_{-}$in $(-\pi, \pi]$. The asymptotic formulas of $\mathbf{M}(a N+1,1, \eta N)$ and $\mathbf{M}^{\prime}(a N+1,1, \eta N)$ can be obtained in the same manner with $n=1$ and $h_{p}(u, \tau)$ replaced by 1 . Thus,

$$
\begin{aligned}
& \mathbf{M}(a N+1,1, \eta N) \sim \frac{(\eta+4 a)^{-1 / 4} e^{N \eta / 2}}{\sqrt{2 \pi N} i(-\eta)^{1 / 4}}\left[e^{i\left(a N \pi-2 a N \varphi_{-}+\frac{1}{2} N \sqrt{-\eta^{2}-4 a \eta}+\frac{3 \pi}{4}-\varphi_{-}\right)}\right. \\
& \left.+e^{-i\left(a N \pi-2 a N \varphi_{-}+\frac{1}{2} N \sqrt{-\eta^{2}-4 a \eta}+\frac{3 \pi}{4}-\varphi_{-}\right)}\right], \\
& \mathbf{M}^{\prime}(a N+1,1, \eta N) \sim \frac{(\eta+4 a)^{-1 / 4} e^{N \eta / 2} \sqrt{a}}{\sqrt{2 \pi N} i(-\eta)^{3 / 4}}\left[e^{i\left(a N \pi-2 a N \varphi_{-}+\frac{1}{2} N \sqrt{-\eta^{2}-4 a \eta}+\frac{3 \pi}{4}-2 \varphi_{-}\right)}\right. \\
& \left.+e^{-i\left(a N \pi-2 a N \varphi_{-}+\frac{1}{2} N \sqrt{-\eta^{2}-4 a \eta}+\frac{3 \pi}{4}-2 \varphi_{-}\right)}\right] .
\end{aligned}
$$

A combination of the last three equations gives the error estimate in (6.16).

Case (ii) Here, $N^{2 / 3}(\eta+4 a)$ is bounded. Hence, $\eta \rightarrow-4 a$ and, correspondingly, $a \rightarrow a_{-}$; see the statement preceding (3.15). For $a<a_{-}$, the contour $\gamma_{u}$ in (6.15) is shown in Figure 7, for $a>a_{-}$, the contour $\gamma_{u}$ is depicted in Figure 6. We divide $\gamma_{u}$ into two parts, so that the error term $\epsilon_{p}^{+}$in (6.15) can be written correspondingly as $\epsilon_{p}^{+} \equiv \epsilon_{p, 1}^{+}+\epsilon_{p, 2}^{+}$. We call the part of $\gamma_{u}$ in the upper half of the plane $\gamma_{u}^{(1)}$, and the part of $\gamma_{u}$ in the lower 
half of the plane $\gamma_{u}^{(2)}$. Because of the cut in the interval $(0,1)$, the phase function $\psi(u)$ of the $u$-integral in $(6.15)$ can be written as

$$
\psi(u)=a \ln u-a \ln (1-u)+\eta u-i a \pi \equiv \widehat{\psi}(u)-i a \pi
$$

for $u \in \gamma_{u}^{(1)}$, and

$$
\psi(u)=\widehat{\psi}(u)+i a \pi
$$

for $u \in \gamma_{u}^{(2)}$. Since $\widehat{\psi}(u)$ has two coalescing saddle points $u_{ \pm}$given in (3.14), we make the standard transformation

$$
\widehat{\psi}(u)=\frac{1}{3} v^{3}-\zeta v+A
$$

with the correspondence of the saddle points $u_{ \pm}$and $\pm \sqrt{\zeta}$ given by

$$
u_{-} \leftrightarrow \sqrt{\zeta}, \quad u_{+} \leftrightarrow-\sqrt{\zeta}
$$

If $\zeta>0$, then $\pm \sqrt{\zeta}$ are both real; if $\zeta<0$, then $\pm \sqrt{\zeta}$ are complex conjugates and purely imaginary. The values of $A$ and $\zeta$ can be obtained by using (7.1) and (7.2). The images of the contour $\gamma_{2}^{(2)}$ under the mapping $u \rightarrow v$ defined in (7.1) are depicted in Figures 13 and 14. The part $\epsilon_{p, 1}^{+}$of the error term $\epsilon_{p}^{+}$now becomes

$$
\epsilon_{p, 1}^{+}=\frac{e^{-i a \pi N+\frac{1}{2} \eta N}}{2 \pi i \eta N^{p+1}} \int_{-\infty}^{\infty} \int_{C_{2}} g(v, \tau) e^{N\left(\frac{1}{3} v^{3}-\zeta v\right)} e^{-N \tau^{2}} \mathrm{~d} v \mathrm{~d} \tau
$$

where

$$
g(v, \tau)=h_{p}(u, \tau) \frac{1}{u-1} \frac{\mathrm{d} u}{\mathrm{~d} v}
$$

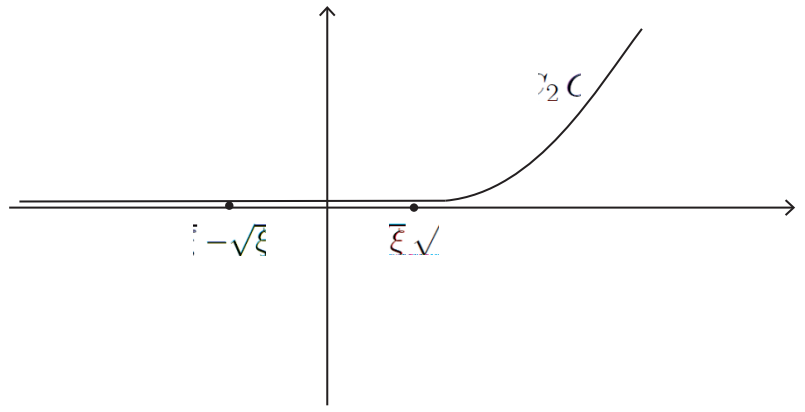

Figure 13: Contour $C_{2}\left(a<a_{-}\right)$.

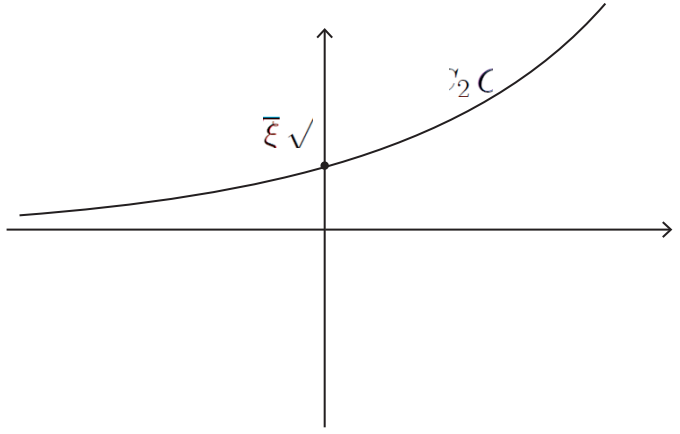

Figure 14: Contour $C_{2}\left(a>a_{-}\right)$.

The part $\epsilon_{p, 2}^{+}$can be handled in exactly the same manner, and we have

$$
\epsilon_{p, 2}^{+}=\frac{e^{i a \pi N+\frac{1}{2} \eta N}}{2 \pi i \eta N^{p+1}} \int_{-\infty}^{\infty} \int_{C_{3}} g(v, \tau) e^{N\left(\frac{1}{3} v^{3}-\zeta v\right)} e^{-N \tau^{2}} \mathrm{~d} v \mathrm{~d} \tau,
$$

where the contour $C_{3}$ is the reflection of $C_{2}$ in the $v$-plane with respect to the real axis. Since the two contours $C_{2}$ and $C_{3}$ together are equivalent to a contour running from 
$\infty e^{-i \pi / 3}$ to $\infty e^{i \pi / 3}$ in the right half-plane, in terms of Airy integral $\mathrm{Ai}(\cdot)$ we have by the Bleistein method [21, p.366-370]

$$
\begin{aligned}
\epsilon_{p}^{+} \sim \operatorname{Re}\left\{\frac{e^{-i a \pi N+\frac{1}{2} \eta N}}{\sqrt{\pi} i \eta N^{p+3 / 2}}[\right. & p_{0} e^{\frac{2}{3} \pi i} N^{-\frac{1}{3}} \operatorname{Ai}\left(N^{\frac{2}{3}} \zeta e^{\frac{2}{3} \pi i}\right) \\
& \left.\left.-q_{0} e^{\frac{4}{3} \pi i} N^{-\frac{2}{3}} \operatorname{Ai}^{\prime}\left(N^{\frac{2}{3}} \zeta e^{\frac{2}{3} \pi i}\right)\right]\right\},
\end{aligned}
$$

where

$$
\begin{aligned}
& p_{0}=\frac{1}{2}[g(\sqrt{\zeta}, 0)+g(-\sqrt{\zeta}, 0)] \\
& q_{0}=\frac{1}{2 \sqrt{\zeta}}[g(\sqrt{\zeta}, 0)-g(-\sqrt{\zeta}, 0)] .
\end{aligned}
$$

For a detailed discussion of uniform asymptotic evaluation of integrals, see [21, Chap.7].

Following the same argument, one can derive the asymptotic approximations of the confluent hypergeometric functions $\mathbf{M}(a N+1,1, \eta N)$ and $\mathbf{M}^{\prime}(a N+1,1, \eta N)$. Indeed, we have

$$
\begin{aligned}
\mathbf{M}(a N+1,1, \eta N) \sim \operatorname{Re}\left\{\frac{e^{-i a \pi N+\frac{1}{2} \eta N}}{\pi i}\right. & {\left[p_{0}^{*} e^{\frac{2}{3} \pi i} N^{-\frac{1}{3}} \mathrm{Ai}\left(N^{\frac{2}{3}} \zeta e^{\frac{2}{3} \pi i}\right)\right.} \\
& \left.\left.-q_{0}^{*} e^{\frac{4}{3} \pi i} N^{-\frac{2}{3}} \mathrm{Ai}^{\prime}\left(N^{\frac{2}{3}} \zeta e^{\frac{2}{3} \pi i}\right)\right]\right\},
\end{aligned}
$$

where $\zeta$ is again determined by (7.1) and (7.2), and is the same as that appearing in (7.4). The coefficients $p_{0}^{*}$ and $q_{0}^{*}$ are similar to those given in (17.5) and (7.6), except that the function $h_{p}(u, \tau)$ in (7.3) is replaced by 1 . The asymptotic formula of $\mathbf{M}^{\prime}(a N+1,1, \eta N)$ is the same as (7.7), only with $h_{p}(u, \tau)$ in (7.3) now replaced by $u$.

A comparison of (17.4) with (77.7) and a corresponding formula for $\mathbf{M}^{\prime}(a N+1,1, \eta N)$ again establishes (6.16).

Case (iii) In the present case, the contour $\gamma_{u}$ in (6.15) is shown in Figure 7. As in case (ii), we divide $\gamma_{u}$ into two parts, and label the parts above and below the real axis by $\gamma_{u}^{(1)}$ and $\gamma_{u}^{(2)}$, respectively. Since $\gamma_{u}^{(1)}$ and $\gamma_{u}^{(2)}$ are symmetric with repect to the real axis and $h_{p}(\bar{u}, \bar{\tau})=\overline{h_{p}(u, \tau)}$, the error term $\epsilon_{p}^{+}$can be written as

$$
\epsilon_{p}^{+}=\frac{1}{\pi \eta N^{p+1}} \operatorname{Im}\left(\int_{-\infty}^{\infty} \int_{\gamma_{u}^{(2)}} \frac{h_{p}(u, \tau)}{u-1} e^{N \psi(u)} e^{-N \tau^{2}} \mathrm{~d} u \mathrm{~d} v\right) .
$$

In view of the equation preceding (17.1), we can further write

$$
\begin{aligned}
\epsilon_{p}^{+}=\frac{1}{\pi \eta N^{p+1}}\{ & \cos (a \pi N) \operatorname{Im}\left(\int_{-\infty}^{\infty} \int_{\gamma_{u}^{(2)}} \frac{h_{p}(u, \tau)}{u-1} e^{N \widehat{\psi}(u)} e^{-N \tau^{2}} \mathrm{~d} u \mathrm{~d} v\right) \\
& \left.+\sin (a \pi N) \operatorname{Re}\left(\int_{-\infty}^{\infty} \int_{\gamma_{u}^{(2)}} \frac{h_{p}(u, \tau)}{u-1} e^{N \widehat{\psi}(u)} e^{-N \tau^{2}} \mathrm{~d} u \mathrm{~d} v\right)\right\},
\end{aligned}
$$


where $\widehat{\psi}(u)=a \ln u-a \ln (1-u)+\eta u$.

Now observe that since $N^{\frac{2}{3}}(\eta+4 a)$ is unbounded, the rate in which $\eta \rightarrow-4 a$ is controlled; that is, the two saddle point $u_{ \pm}$of the phase function $\widehat{\psi}(u)$ do not coalesce sufficiently fast; cf. (3.14). As a consequence, it is known that the classical method of steepest descent continues to apply. (Note that $N \widehat{\psi}(u)=N(a \ln u-a \ln (1-u)+\eta u)$. So, to apply this method, $a N$ must approach infinity; i.e., $x$ is large.)

In (7.8), there are two terms, and both terms contain the same double integral. Since $\widehat{\psi}(u)$ is real for $u \in(0,1)$, to get the real part of the asymptotic approximation of the double integral we need be concerned with only the horizontal part of the contour $\gamma_{u}^{(2)}$. Furthermore, since $\widehat{\psi}\left(u_{+}\right)>\widehat{\psi}\left(u_{-}\right)$, the real part of the double integral in (7.8) is asymptotically equal to

$$
\frac{h_{p}\left(u_{+}, 0\right)}{u_{+}-1} e^{N \widehat{\psi}\left(u_{+}\right)} \sqrt{\frac{2}{-\widehat{\psi}^{\prime \prime}\left(u_{+}\right)}} \frac{\pi}{N} .
$$

Since the integrand on the horizontal part of the contour $\gamma_{u}^{(2)}$ is real, only the curved part of contour $\gamma_{u}^{(2)}$ contributes to the imaginary part of the double integral. Since the cut for $\widehat{\psi}(u)$ is now from 1 to $+\infty$, we can extend the contour $\gamma_{u}^{(2)}$ into the upper half-plane by its mirror image, and form the integration path $\Gamma$ which passes through $u_{-}$as an interior point. The imaginary part of the double integral in (7.8) is asymptotically equal to

$$
\frac{1}{2 i} \frac{h_{p}\left(u_{-}, 0\right)}{u_{-}-1} e^{N \widehat{\psi}\left(u_{-}\right)} \sqrt{\frac{2}{-\widehat{\psi}^{\prime \prime}\left(u_{-}\right)}} \frac{\pi}{N} .
$$

Inserting the contributions given in (7.9) and (7.10) into equation (7.8) gives

$$
\begin{aligned}
\epsilon_{p}^{+}=\frac{1}{\eta N^{p+2}}\{ & \sin (a \pi N) \frac{h_{p}\left(u_{+}, 0\right)}{u_{+}-1} e^{N \widehat{\psi}\left(u_{+}\right)} \sqrt{\frac{2}{-\widehat{\psi}^{\prime \prime}\left(u_{+}\right)}}\left[1+O\left(N^{-1}\right)\right] \\
& \left.+\frac{\cos (a \pi N)}{2 i} \frac{h_{p}\left(u_{-}, 0\right)}{u_{-}-1} e^{N \widehat{\psi}\left(u_{-}\right)} \sqrt{\frac{2}{-\widehat{\psi}^{\prime \prime}\left(u_{-}\right)}}\left[1+O\left(N^{-1}\right)\right]\right\} .
\end{aligned}
$$

Again, since $\widehat{\psi}\left(u_{+}\right)>\widehat{\psi}\left(u_{-}\right)$, (7.11) implies

$$
\begin{aligned}
\epsilon_{p}^{+}=\frac{e^{N \widehat{\psi}\left(u_{+}\right)}}{\eta N^{p+2}}\{ & \sin (a \pi N) \frac{h_{p}\left(u_{+}, 0\right)}{u_{+}-1} \sqrt{\frac{2}{-\widehat{\psi}^{\prime \prime}\left(u_{+}\right)}}\left[1+O\left(N^{-1}\right)\right] \\
& + \text { an exponentially small term }\} .
\end{aligned}
$$

Asymptotic approximations for $\mathbf{M}(a N+1,1, \eta N)$ and $\mathbf{M}^{\prime}(a N+1,1, \eta N)$ can be obtained in a similar manner, and we have

$$
\begin{aligned}
\mathbf{M}(a N+1,1, \eta N)=\frac{e^{N \widehat{\psi}\left(u_{+}\right)}}{\sqrt{\pi N}}\left\{\frac{\sin (a \pi N)}{u_{+}-1} \sqrt{\frac{2}{-\widehat{\psi}^{\prime \prime}\left(u_{+}\right)}}\left[1+O\left(N^{-1}\right)\right]\right. \\
+ \text { an exponentially small term }\}
\end{aligned}
$$




$$
\begin{aligned}
\mathbf{M}^{\prime}(a N+1,1, \eta N)=\frac{e^{N \widehat{\psi}\left(u_{+}\right)}}{\sqrt{\pi N}}\{ & \sin (a \pi N) \frac{u_{+}}{u_{+}-1} \sqrt{\frac{2}{-\widehat{\psi}^{\prime \prime}\left(u_{+}\right)}}\left[1+O\left(N^{-1}\right)\right] \\
& + \text { an exponentially small term }\} .
\end{aligned}
$$

The estimate in (6.16) now follows from (7.12), (7.13) and (7.14).

Remark. To be more precise, the exponentially small term in (7.13) and (7.14) is $O\left(e^{N\left(\widehat{\psi}\left(u_{-}\right)-\widehat{\psi}\left(u_{+}\right)\right.}\right)$.

Case (iv) Here, we proceed as in case (iii), and reach the error representation given in (7.8). There are two terms in this equation; we begin with the one involving

$$
G_{2} \equiv \operatorname{Re}\left(\int_{-\infty}^{\infty} \int_{\gamma_{u}^{(2)}} \frac{h_{p}(u, \tau)}{u-1} e^{N \widehat{\psi}(u)} e^{-N \tau^{2}} \mathrm{~d} u \mathrm{~d} \tau\right) .
$$

As explained in the previous case, for this term we need be concerned with only the horizontal part of the contour $\gamma_{u}^{(2)}$. Since $a N$ is bounded, $a$ must tend to zero and $\eta$ is negative; see the correspondence between $a$ and $\eta$ given in 93 . Thus, the saddle point $u_{ \pm}$ behave like

$$
\begin{aligned}
& u_{+}=\frac{1}{2}-\frac{1}{2} \sqrt{1+\frac{4 a}{\eta}}=-\frac{a}{\eta}+O\left(a^{2}\right) \rightarrow 0, \\
& u_{-}=\frac{1}{2}+\frac{1}{2} \sqrt{1+\frac{4 a}{\eta}}=1+\frac{a}{\eta}+O\left(a^{2}\right) \rightarrow 1 .
\end{aligned}
$$

As $u \rightarrow 0, \widehat{\psi}(u) \sim a \ln u+(a+\eta) u$. This suggests that we make the transformation

$$
\widehat{\psi}(u)=a \ln v+(a+\eta) v \equiv \rho(v)
$$

with $u=u_{+}$corresponding to $v=v_{0}=-\frac{a}{a+\eta}$. Recall that $\eta$ is negative and $a \rightarrow 0$; thus, $v_{0}>0$ and $v_{0} \rightarrow 0$. This says that our saddle point $v_{0}$ coalesces with an endpoint. We also note that $N \widehat{\psi}(u)=N a \ln v+N(a+\eta) v$, and that $a N$ is bounded. All these point out that the steepest descent method does not apply. Therefore, we rewrite (7.15) as

$$
G_{2} \sim \operatorname{Re}\left(\int_{-\infty}^{\infty} \int_{0}^{+\infty} g(v, \tau) v^{a N} e^{N(a+\eta) v} e^{-N \tau^{2}} \mathrm{~d} v \mathrm{~d} \tau\right)
$$

where

$$
g(v, \tau)=\frac{h_{p}(u, \tau)}{u-1} \frac{\mathrm{d} u}{\mathrm{~d} v}
$$

Since

$$
\left.\frac{\mathrm{d} u}{\mathrm{~d} v}\right|_{v=v_{0}}=\sqrt{\frac{\rho^{\prime \prime}\left(v_{0}\right)}{\widehat{\psi}^{\prime \prime}\left(u_{+}\right)}}=\left.\sqrt{\frac{u^{2}(u-1)^{2}}{v^{2}(1-2 u)}}\right|_{u=u_{+}, v=v_{0}} \rightarrow 1
$$

as $a \rightarrow 0$, we obtain by Watson's lemma

$$
G_{2}=-\frac{h_{p}(0,0)}{[-N(a+\eta)]^{a N+1}} \sqrt{\frac{\pi}{N}} \Gamma(a N+1)\left[1+O\left(N^{-1}\right)\right] .
$$


Next, we consider the first term in (7.8)

$$
G_{1} \equiv \operatorname{Im}\left(\int_{-\infty}^{\infty} \int_{\gamma_{u}^{(2)}} \frac{h_{p}(u, \tau)}{u-1} e^{N \widehat{\psi}(u)} e^{-N \tau^{2}} \mathrm{~d} u \mathrm{~d} \tau\right)
$$

As in case (iii), since the integrand on the horizontal part of the contour $\gamma_{u}^{(2)}$ is real, by symmetry we have

$$
G_{1}=\frac{1}{2 i}\left(\int_{-\infty}^{\infty} \int_{L} \frac{h_{p}(u, \tau)}{u-1} e^{N \widehat{\psi}(u)} e^{-N \tau^{2}} \mathrm{~d} u \mathrm{~d} \tau\right)
$$

where $L$ is the curved part of $\gamma_{u}^{(2)}$ plus its minor image in the upper half-plane. Recall that there is a cut for $\widehat{\psi}(u)$, which extends from $u=1$ to $u=+\infty$ along the positive real line. Since $u_{-}=1+\frac{a}{\eta}+\cdots \sim 1$ as $a \rightarrow 1$, this saddle point coalesces with the branch point at $u=1$. For $u$ near 1 , we write $u=1-v$ with $v$ being small. Then,

$$
\widehat{\psi}(u) \sim-a \ln v+\eta-(\eta+a) v .
$$

This suggests the transformation

$$
\widehat{\psi}(u)=-a \ln z+\eta-(\eta+a) z \equiv \widehat{\rho}(z) .
$$

The double integral in (7.20) now becomes

$$
G_{1}=\frac{1}{2 i} \int_{-\infty}^{\infty} \int_{-\infty}^{\left(0^{+}\right)} \frac{h_{p}(u, \tau)}{u-1} \frac{\mathrm{d} u}{\mathrm{~d} z} e^{N \widehat{\psi}(u)} e^{-N \tau^{2}} \mathrm{~d} z \mathrm{~d} \tau .
$$

Since $N \widehat{\rho}(z)=N a \ln z-N(\eta+a) z+N \eta$ and $N a$ is bounded, the method of steepest descent does not apply. So we rewrite (17.22) in the form

$$
G_{1}=\frac{1}{2 i} e^{N \eta} \int_{-\infty}^{\infty} \int_{-\infty}^{\left(0^{+}\right)} z^{-a N-1} g(z, \tau) e^{-N(\eta+a) z} e^{-N \tau^{2}} \mathrm{~d} z \mathrm{~d} \tau,
$$

where

$$
g(z, \tau)=\frac{z}{u-1} \frac{\mathrm{d} u}{\mathrm{~d} z} h_{p}(u, \tau) .
$$

Recall that $N(\eta+a)$ is negative. To the inner integral, we can use Watson's Lemma for loop integrals [21, p.20]. Since

$$
\left.\frac{z}{u-1}\right|_{z=0, u=1}=-1 \quad \text { and }\left.\quad \frac{\mathrm{d} u}{\mathrm{~d} z}\right|_{z=0, u=1}=-1,
$$

we have $g(0,0)=h_{p}(1,0)$ and

$$
G_{1}=\pi h_{p}(1,0) \sqrt{\frac{\pi}{N}} \frac{[-N(a+\eta)]^{a N}}{\Gamma(a N+1)} e^{\eta N}\left[1+O\left(N^{-1}\right)\right] .
$$

Coupling (7.18) and (7.23), we have from (7.8)

$$
\begin{aligned}
\epsilon_{p}^{+}=\frac{1}{\eta N^{p+1}}\{ & \cos (a N \pi) h_{p}(1,0) \sqrt{\frac{\pi}{N}} \frac{[-N(a+\eta)]^{a N}}{\Gamma(a N+1)} e^{\eta N}\left[1+O\left(N^{-1}\right)\right] \\
& \left.-\sin (a N \pi) h_{p}(0,0) \sqrt{\frac{\pi}{N}} \frac{\Gamma(a N+1)}{\pi[-N(a+\eta)]^{a N+1}}\left[1+O\left(N^{-1}\right)\right]\right\}
\end{aligned}
$$


which in turn gives

$$
\begin{aligned}
\epsilon_{p}^{+}=\frac{1}{\eta N^{p+1}}\{ & -\frac{\sin (a N \pi) h_{p}(0,0)}{\pi[-N(a+\eta)]^{a N+1}} \sqrt{\frac{\pi}{N}} \Gamma(a N+1)\left[1+O\left(N^{-1}\right)\right] \\
& \left.+O\left(e^{N \eta}\right)\right\} .
\end{aligned}
$$

Note that $\eta$ is negative.

The asymptotics of $\mathbf{M}(a N+1,1, \eta N)$ and $\mathbf{M}^{\prime}(a N+1,1, \eta N)$ can again be obtained in a similar manner. We have

$$
\begin{aligned}
\mathbf{M}(a N+1,1, \eta N)= & -\frac{\sin (a N \pi)}{\pi[-N(a+\eta)]^{a N+1}} \Gamma(a N+1)\left[1+O\left(N^{-1}\right)\right] \\
& +O\left(e^{N \eta}\right), \\
\mathbf{M}^{\prime}(a N+1,1, \eta N)= & \frac{a \sin (a N \pi)}{\pi \eta[-N(a+\eta)]^{a N+1}} \Gamma(a N+1)\left[1+O\left(N^{-1}\right)\right] \\
& +O\left(e^{N \eta}\right) .
\end{aligned}
$$

The estimate (6.16) follows immediately from (7.24), (7.25) and (7.26).

Case (v) We first consider the subcase in which $a$ is bounded; that is, $a \in(-M, 0)$, where $M>0$ is a constant. Since in this case only the saddle point $u=a$ matters (see $(3.26)$ ), by applying the steepest descent method directly, we obtain

$$
\epsilon_{p}^{-}=\frac{h_{p}(a, 0)}{N^{p+1 / 2}} \sqrt{\pi}\left[1+O\left(N^{-1}\right)\right] .
$$

Since $h_{p}(u, \tau)$ is analytic along the path of integration, for bounded $a$ we can easily find a constant $M_{p}$ satisfying (6.24).

Next, we consider the subcase: $a \rightarrow-\infty$. Motivated by a method of Olde Daalhuis and Temme [22], we introduce the rational functions

$$
\begin{aligned}
R_{0}(u, w, a) & =\frac{1}{u-w} \\
R_{n+1}(u, w, a) & =\frac{-1}{u-a} \frac{\mathrm{d}}{\mathrm{d} u}\left(u R_{n}(u, w, a)\right), \quad n=0,1,2, \cdots,
\end{aligned}
$$

where $u, w \in \mathbb{C}, u \neq w$ and $u^{2} \neq a^{2}$. Using an induction argument on $n$, it can be readily verified that there are constants $C_{i j}$, independent of $u, w$ and $a$, such that

$$
R_{n}(u, w, a)=w \sum_{i=0}^{n-1} \sum_{j=0}^{n-1-i} \frac{C_{i j} u^{n-1-j}}{(u-w)^{n+1-i-j}(u-a)^{n+i}}, \quad n=1,2, \cdots
$$

To extend the validity of the asymptotic expansion (6.21) from bounded $a$ to unbounded $a$, we first define

$$
\rho_{0}(a)=\min \left\{|u-a|: u \text { is a singularity of } h_{0}(u)\right\}
$$


Since we shall be interested in the property of $h_{p}(u, \tau)$ in $u$, we may suppress the dependence on $\tau$ and simply write $h_{p}(u)$ for $h_{p}(u, \tau)$. To obtain an estimate on $\rho_{0}(a)$, we denote by $u_{ \pm k}$ the singular points in the $u$-plane, which are the images of $w_{-} e^{ \pm 2 k \pi i}$ under the mapping (3.27)-(3.28), where $w_{-}$is the saddle point given in (3.9). Thus, we have

$$
\begin{aligned}
\tilde{f}\left(t_{0}\left(w_{-}\right), w_{-}\right) & =a \ln (-a)-a+\gamma \\
\widetilde{f}\left(t_{0}\left(w_{-} e^{ \pm 2 k \pi i}\right), w_{-} e^{ \pm 2 k \pi i}\right) & =a \ln \left(-u_{ \pm k}\right)-u_{ \pm k}+\gamma .
\end{aligned}
$$

Subtracting (7.32) from (7.33) gives

$$
\pm 2 k \pi(a+b-1) i=a \ln \left(-u_{ \pm k}\right)-u_{ \pm k}-a \ln (-a)+a .
$$

For large values of $a$, numerical calculation shows that the nearest singularities should be $u_{ \pm 1}$ which are approximately equal to $(3.089 \pm 7.461 i) a$. Hence $\rho_{0}(a) \sim-7.75 a$. (Recall that $a$ is negative). [Put $u_{ \pm k}=a v_{ \pm k}$ in (7.34), and have

$$
\pm 2 k \pi\left(\frac{a+b-1}{a}\right) i=\ln \left(v_{ \pm k}\right)-v_{ \pm k}+1 \text {. }
$$

For large $a$, the left-hand side is approximately equal to $\pm 2 k \pi i$. Numerical computation of the resulting equation gives $v_{ \pm 1}=3.089 \pm 7.461 i$.]

To estimate the error term $\varepsilon_{p}^{-}$in (6.23), we split the loop contour into two parts $\mathcal{L}_{1}$ and $\mathcal{L}_{2}$, and write

$$
\begin{aligned}
& \varepsilon_{p, 1}^{-}=\frac{N^{a N} \Gamma(-a N+1)}{2 \pi i N^{p}} \int_{-\infty}^{+\infty} \int_{\mathcal{L}_{1}} \frac{h_{p}(u, \tau)}{u} e^{N \widetilde{\psi}(u)} e^{-N \tau^{2}} \mathrm{~d} u \mathrm{~d} \tau \\
& \varepsilon_{p, 2}^{-}=\frac{N^{a N} \Gamma(-a N+1)}{2 \pi i N^{p}} \int_{-\infty}^{+\infty} \int_{\mathcal{L}_{2}} \frac{h_{p}(u, \tau)}{u} e^{N \widetilde{\psi}(u)} e^{-N \tau^{2}} \mathrm{~d} u \mathrm{~d} \tau
\end{aligned}
$$

where $\mathcal{L}_{1}=\left\{u \in\right.$ loop contour : $\left.|u-a| \leq c|a|^{\theta}\right\}$, with $0<c<1$ and $\frac{3}{4} \leq \theta \leq 1$. A simple estimation shows that $\varepsilon_{p, 2}^{-}$is exponentially small in comparison with $\varepsilon_{p, 1}^{-}$; see [22, p. 318-320].

Let $\Gamma_{1}$ be a closed contour embracing the curve $\mathcal{L}_{1}$ such that as $a \rightarrow-\infty$,

$$
\text { length } \Gamma_{1}=O\left(|a|^{\theta}\right) \quad \text { and } \quad \text { distance }\left(\Gamma_{1}, \mathcal{L}_{1}\right) \sim c_{1}|a|^{\theta} \text {. }
$$

Let $\Omega_{1}$ denote the domain bounded by $\Gamma_{1}$, and its closure by $\bar{\Omega}_{1}$. Similarly, let $\Gamma_{2}$ be a closed curve embracing $\mathcal{L}_{1}$ and lying inside $\Gamma_{1}$ such that as $a \rightarrow-\infty$,

$$
\text { length } \Gamma_{2}=O\left(|a|^{\theta}\right) \quad \text { and } \quad \text { distance }\left(\Gamma_{2}, \mathcal{L}_{1}\right) \sim c_{2}|a|^{\theta}
$$

with $c_{2}<c_{1}$. Let $\Omega_{2}$ denote the domain bounded by $\Gamma_{2}$, and its closure by $\bar{\Omega}_{2}$. Define

$$
\widetilde{h}_{p}=\sup _{w \in \bar{\Omega}_{1}}\left|h_{p}(w)\right|
$$

It is easily verified that as $a \rightarrow-\infty$

$$
\sup _{w \in \bar{\Omega}_{1}}\left|h_{0}(w)\right| \leq c_{0}\left|h_{0}(a)\right|
$$


where $c_{0}$ is another constant independent of $a$. From (7.30), we also have

$$
\sup _{u \in \Gamma_{1}, w \in \bar{\Omega}_{2}}\left|R_{p}(u, w, a)\right| \leq C_{p}|a|^{-(2 \theta-1) p-\theta},
$$

where $C_{p}$ is a constant independent of $a$. By (7.40), (7.41) and (7.37), it follows that

$$
\left|\frac{1}{2 \pi i} \int_{\Gamma_{1}} R_{p}(u, w, a) h_{0}(u) \mathrm{d} u\right| \leq C_{p}^{\prime}|a|^{-(2 \theta-1) p}\left|h_{0}(a)\right|,
$$

where $C_{p}^{\prime}$ is also a constant. By Cauchy's integral formula and (6.20b) and (7.29), we have upon integration by parts

$$
\begin{aligned}
h_{p}(w) & =\frac{1}{2 \pi i} \int_{\Gamma_{1}} R_{0}(u, w, a) h_{p}(u) \mathrm{d} u \\
& =-\frac{1}{2 \pi i} \int_{\Gamma_{1}} g_{p-1}(u) \mathrm{d}\left(u R_{0}(u, w, a)\right) \\
& =\frac{1}{2 \pi i} \int_{\Gamma_{1}}(u-a) R_{1}(u, w, a) g_{p-1}(u) \mathrm{d} u \\
& =\frac{1}{2 \pi i} \int_{\Gamma_{1}} R_{1}(u, w, a) h_{p-1}(u) \mathrm{d} u-\frac{1}{2 \pi i} \int_{\Gamma_{1}} a_{p-1} R_{1}(u, w, a) \mathrm{d} u
\end{aligned}
$$

where we have used (6.20a) to get $h_{p-1}(a, \tau)=a_{p-1}(\tau)$. On account of (7.41), the last integral is $\widetilde{h}_{p-1}(u) O\left(|a|^{-(2 \theta-1)}\right)$. Repeated application of (1.43) gives

$$
h_{p}(w)=\frac{1}{2 \pi i} \int_{\Gamma_{1}} R_{p}(u, w, a) h_{0}(u) \mathrm{d} u+\widetilde{h}_{p-1} O\left(|a|^{-(2 \theta-1)}\right)+\cdots+\widetilde{h}_{0} O\left(|a|^{-(2 \theta-1) p}\right)
$$

By induction, we have from (7.44) and (7.42)

$$
\widetilde{h}_{p} \leq \widetilde{C}_{p}|a|^{-(2 \theta-1) p}\left|h_{0}(a)\right|,
$$

where $\widetilde{C}_{p}$ is a constant independent of $a$. Substituting (17.45) in (7.35), we obtain

$$
\left|\varepsilon_{p, 1}^{-}\right| \leq \frac{A_{p}|a|^{-(2 \theta-1) p}\left|h_{0}(a)\right| \sqrt{\pi}}{N^{p+1 / 2}}
$$

where $A_{p}$ is a constant not depending on $a$. From (5.3), one can show that $h_{0}(a)=$ $h_{0}(a, \tau) \sim \sqrt{-a}$ as $a \rightarrow-\infty$. Thus, to have the right-hand side of (7.46) independent of $a$, we must choose $\theta$ so that $(2 \theta-1) \geq \frac{1}{2 p} \geq \frac{1}{2}$ for all $p \geq 1$; that is, $\theta \geq \frac{3}{4}$. With this choice, we can always find a constant $M_{p}$, independent of $a$, for each $p$ such that (6.24) holds.

\section{SUMMARY OF RESULTS}

For $a<0$, we have from (6.21) and (6.24)

$$
t_{n}(x, N+1) \sim \frac{(-1)^{n} \Gamma(n+N+2) N^{-a N} e^{N \gamma}}{\Gamma(n+1) \Gamma(N-n+1) \Gamma(-a N+1)} \sum_{l=0}^{\infty} \frac{c_{l}}{N^{l+\frac{1}{2}}}
$$


as $N \rightarrow \infty$, where the coefficients $c_{l}$ are given in (6.22) and the constant $\gamma$ is determined by (3.27) and (3.28).

For $0 \leq a \leq \frac{1}{2}$, we have from (6.12) and (6.16)

$$
\begin{aligned}
t_{n}(x, N+1) \sim \frac{(-1)^{n} \Gamma(n+N+2) e^{N \gamma}}{\Gamma(n+1) \Gamma(N-n+1)}\left[\mathbf{M}(a N+1,1, \eta N) \sum_{l=0}^{\infty} \frac{c_{l}}{N^{l+\frac{1}{2}}}\right. \\
\left.+\mathbf{M}^{\prime}(a N+1,1, \eta N) \sum_{l=0}^{\infty} \frac{d_{l}}{N^{l+\frac{1}{2}}}\right]
\end{aligned}
$$

as $N \rightarrow \infty$, where $\mathbf{M}$ is the confluent hypergeometric function defined in (6.4), and $\mathbf{M}^{\prime}$ is its derivative taken with respect to the third variable. (Note that the second variable in $\mathbf{M}$ is a constant.) The coefficients $c_{l}$ and $d_{l}$ in (8.2) are given in (6.13) and (6.14), respectively. The constants $\eta$ and $\gamma$ are determined by (3.15) and (3.16).

For $a>\frac{1}{2}$, the asymptotic expansion of $t_{n}(x, N+1)$ can be obtained by using the symmetry relation (1.8),

$$
t_{n}(a N, N+1)=(-1)^{n} t_{n}((1-a) N, N+1) .
$$

The regions of validity of the expansions (8.1) and (8.2) are, respectively, $a<0$ and $0 \leq a \leq \frac{1}{2}$. However, as we shall show in $\$ 9$, they can be extended to two overlapping regions both containing a neighborhood of $a=0$.

The integral given in (3.12) for the confluent hypergeometric function is a typical example of Airy-type expansion [21, Chap. VII, §4]. In fact, we have already illustrated this point in (7.7), and the region of validity of this formula is $0<\delta \leq a \leq \frac{1}{2}$. Thus, for $\delta \leq a \leq \frac{1}{2}$, one can derive from (8.2) an asymptotic approximation for $t_{n}(x, N+1)$ in terms of the Airy integral $\mathrm{Ai}(\cdot)$ and its derivative $\mathrm{Ai}^{\prime}(\cdot)$.

We now derive from (8.1) and (8.2) two simple formulas for $t_{n}(x, N+1)$ when $x$ is fixed, which in turn means $a=O(1 / N)$. First, we consider the case when $x$ is negative, i.e., $a<0$. To calculate the leading coefficient $c_{0}$ in (8.1), we use (6.22), (6.20c) and (6.20a). Thus, we have $c_{0}=a_{0,0} \Gamma\left(\frac{1}{2}\right)$ and $a_{0,0}=a_{0}(0)=h_{0}(a, 0)$. From (5.3), it can be readily verified that

$$
h(a, 0)=-\frac{\sqrt{2(1-b)}}{b^{3 / 2}} a+O\left(a^{2}\right) \quad \text { as } a \rightarrow 0^{-} .
$$

Hence,

$$
c_{0}=-\frac{\sqrt{2(1-b) \pi}}{b^{3 / 2}} a+O\left(a^{2}\right) \quad \text { as } a \rightarrow 0^{-} .
$$

To find the value of the constant $\gamma$ in (8.1), we recall (3.27) and (3.28), and obtain

$$
\gamma=\tilde{f}\left(t_{0}\left(w_{-}\right), w_{-}\right)-a \ln (-a)+a .
$$

Since $t_{0}\left(w_{-}\right)=t_{-}$, from (2.10) and (3.2) it follows that

$$
\gamma=b \ln b+(1-b) \ln (1-b)-2 a \ln b+O\left(a^{2}\right) \quad \text { as } a \rightarrow 0^{-} .
$$


Inserting (8.4) and (8.5) in (8.1) gives

$$
t_{n}(x, N+1)=\frac{(-1)^{n+1} \Gamma(n+N+2) N^{x} n^{-2 x-2}}{\Gamma(N+1) \Gamma(-x)}\left[1+O\left(\frac{1}{N}\right)\right]
$$

for fixed $x<0$.

Next, we consider the case when $x$ is nonnegative; this means that $0 \leq a \leq \frac{1}{2}$ and $a N$ is bounded. Substituting (7.25) and (17.26) in (8.2) gives

$$
\begin{aligned}
t_{n}(x, N+1)= & \frac{(-1)^{n} \Gamma(n+N+2) e^{N \gamma}}{\Gamma(n+1) \Gamma(N-n+1)}\left\{\frac{\Gamma(x+1) \sin \pi x}{\sqrt{N}[-N(a+\eta)]^{a N+1} \pi}\right. \\
& \left.\times\left[-c_{0}+\frac{a}{\eta} d_{0}\right]\left[1+O\left(\frac{1}{N}\right)\right]+O\left(e^{N \eta}\right)\right\} .
\end{aligned}
$$

The coefficients $c_{0}$ and $d_{0}$ can be calculated by using (6.13), (6.14), (3.14) and (6.2); the result is

$$
\begin{aligned}
-c_{0}+\frac{a}{\eta} d_{0} & =-\sqrt{\pi}\left[a_{0,0}-\frac{a}{\eta} b_{0,0}\right] \sim-\sqrt{\pi}\left[a_{0,0}+u_{+} b_{0,0}\right] \\
& =-\sqrt{\pi} h_{0}\left(u_{+}, 0\right) .
\end{aligned}
$$

From (5.2), it can be shown that

$$
h_{0}\left(u_{+}, 0\right)=-\frac{2 \eta \sqrt{1-b}}{b^{3 / 2}}+O(a) \quad \text { as } a \rightarrow 0^{+} .
$$

Hence,

$$
-c_{0}+\frac{a}{\eta} d_{0}=\frac{2 \sqrt{\pi} \eta \sqrt{1-b}}{b^{3 / 2}}+O(a) \quad \text { as } a \rightarrow 0^{+} .
$$

By (3.1) and (3.2), we also have

$$
\begin{aligned}
w_{+} & =1-\frac{a}{b^{2}}+O\left(a^{2}\right), & w_{-} & =\frac{a}{b^{2}}+O\left(a^{2}\right), \\
t_{+} & =\frac{1}{1+b}-\frac{1}{1+b} a+O\left(a^{2}\right), & t_{-} & =(1-b)+(1-b) a+O\left(a^{2}\right) .
\end{aligned}
$$

Inserting (8.10) into (4.2), and subtracting the two resulting equations, we obtain

$$
\begin{aligned}
\eta= & -[(1-b) \ln (1-b)+(1+b) \ln (1+b)] \\
& -2 a \ln \frac{(1-b) \ln (1-b)+(1+b) \ln (1+b)}{b^{2}}+O\left(a^{2}\right) .
\end{aligned}
$$

Solving $\gamma$ in (4.2) also gives

$$
\begin{aligned}
\gamma= & b \ln b+(1-b) \ln (1-b) \\
& +a \ln \frac{(1-b) \ln (1-b)+(1+b) \ln (1+b)}{b^{2}}+O\left(a^{2}\right) .
\end{aligned}
$$

A combination of (8.9), (8.11), (8.12) and (8.7) yields

$$
\begin{aligned}
t_{n}(x, N+1)= & \frac{(-1)^{n+1} \Gamma(n+N+2) N^{x} n^{-2 x-2} \Gamma(x+1)}{\Gamma(N+1) \pi} \\
& \times\left\{\sin \pi x\left[1+O\left(\frac{1}{N}\right)\right]+O\left(e^{N \eta}\right)\right\} ;
\end{aligned}
$$

cf. (1.11). 


\section{REGIONS OF VALIDITY FOR EXPANSIONS (8.1) AND (8.2)}

We first consider the case of expansion (8.1), which is valid for $a<0$. Similarity of the two asymptotic formulas in (8.6) and (8.13) suggests that the region of validity of expansion (8.1) may be extended beyond the origin. This is indeed the case, and we shall show that expansion (8.1) actually holds for $a \in\left(-\infty, a_{-}-\delta\right.$ ] for any small number $\delta>0, a_{-}$being the point on the positive real line given in (3.3), where the two saddle points $w_{ \pm}$coalesce with each other.

To prove this, we recall that when $0 \leq a \leq a_{-}-\delta$, we have $f\left(t_{0}\left(w_{+}\right), w_{+}\right)<$ $f\left(t_{0}\left(w_{-}\right), w_{-}\right)$; see a statement following (3.10). In other words, in the case $0 \leq a \leq a_{-}-\delta$, the contribution from the saddle point $w_{+}$is exponentially small in comparison with that from the saddle point $w_{-}$. So, up to an exponentially small term (E.S.T), we need deal with only one saddle point, namely, $w_{-}$. By an argument similar to that in case (iii) of $\S 7$ (see, especially, equation (7.8)), the integral representation for $t_{n}(x, N+1)$ given in (3.8) can now be written as

$$
\begin{aligned}
t_{n}(x, N+1)= & \frac{(-1)^{n}}{\pi} \frac{\Gamma(n+N+2)}{\Gamma(n+1) \Gamma(N-n+1)} \\
& \times \int_{-\infty}^{+\infty}\left[\sin (a N \pi) \int_{0}^{w_{+}} \frac{1}{w-1} e^{N \widehat{f}\left(t_{0}(w), w\right)} \mathrm{d} w+\text { E.S.T }\right] \frac{\mathrm{d} t}{\mathrm{~d} \tau} e^{-N \tau^{2}} \mathrm{~d} \tau
\end{aligned}
$$

where

$$
\begin{aligned}
\widehat{f}\left(t_{0}(w), w\right)= & b \ln \left(1-t_{0}(w)\right)+(1-b) \ln t_{0}(w) \\
& +a \ln w-a \ln (1-w)+b \ln \left[1-\left(1-t_{0}(w)\right) w\right]
\end{aligned}
$$

which is real on the integration path of $w$. [Note that the term $a \ln (w-1)$ in $f\left(t_{0}(w), w\right)$ is replaced by $a \ln (1-w)$ in $\widehat{f}\left(t_{0}(w), w\right)$.] Similar to (3.27), we make the transformation $w \rightarrow u$ defined by

$$
\widehat{f}\left(t_{0}(w), w\right)=a \ln (u)-u+\gamma
$$

with

$$
u\left(w_{-}\right)=a .
$$

Inserting (9.3) into (9.1) gives

$$
\begin{aligned}
t_{n}(x, N+1)= & \frac{(-1)^{n}}{\pi} \frac{\Gamma(n+N+2)}{\Gamma(n+1) \Gamma(N-n+1)} \int_{-\infty}^{+\infty}\left[\sin (a N \pi) e^{N \gamma}\right. \\
& \left.\times \int_{0}^{\infty} \frac{h(u, \tau)}{u} e^{N(a \ln (u)-u)} \mathrm{d} u+\text { E.S.T }\right] e^{-N \tau^{2}} \mathrm{~d} \tau
\end{aligned}
$$

where

$$
h(u, \tau)=\frac{u}{w-1} \frac{\mathrm{d} w}{\mathrm{~d} u} \frac{\mathrm{d} t}{\mathrm{~d} \tau} .
$$


The derivative $\mathrm{d} w / \mathrm{d} u$ is again given by (3.31) for $u \neq a$ and (3.32) for $u=a$. Let $h_{0}(u, \tau) \equiv h(u, \tau)$, and define the sequence $\left\{h_{l}(u, \tau)\right\}$ as in (6.20b). Following the procedure as in case (ii) of \$匡, we arrive at

$$
\begin{aligned}
t_{n}(x, N+1) \sim & \frac{(-1)^{n} \Gamma(n+N+2)}{\Gamma(n+1) \Gamma(N-n+1)} \\
& \times\left[\frac{N^{-a N} e^{N \gamma} \Gamma(a N) \sin (a N \pi)}{\pi} \sum_{l=0}^{\infty} \frac{c_{l}}{N^{l+\frac{1}{2}}}+\text { E.S.T }\right],
\end{aligned}
$$

where the coefficients $c_{l}$ are as given in (6.22). Although the constant $\gamma$ in (9.7) is determined by the mapping defined by (9.3) and (9.4) while the corresponding constant $\gamma$ in (8.1) is determined by a different mapping defined by (3.27) and (3.28), the two constants are actually the same and can be defined by a single equation

$$
\gamma=\operatorname{Re}\left(\tilde{f}\left(t_{0}\left(w_{-}\right), w_{-}\right)-a \ln (-a)\right)-a,
$$

where $\widetilde{f}\left(t_{0}(w), w\right)$ is the same function given in (3.27) or (2.10). [Recall that $\gamma$ is a real number by Theorem 1 in \$4.] Thus, the two expansions (8.1) and (9.7) are exactly the same, except for an exponentially small term. The region of validity for expansion (8.1) is therefore extended from $(-\infty, 0)$ to $\left(-\infty, a_{-}-\delta\right], 0<\delta<a_{-}$.

Next, we consider the case of expansion (8.2). In the derivation of expansion (8.1) when $a<0$, we have used a mapping $w \rightarrow u$ defined by (3.27). The right-hand side of this equation is the phase function of the Hankel loop integral for Gamma function; see (3.25). But, as an alternative, we can also use the phase function in the integral representation of the confluent hypergeometric function, as in what we have done when $0 \leq a \leq \frac{1}{2}$. This will enable us to extend the validity of expansion (8.2) to negative values of $a$. To prove this, we mention that for $\operatorname{Rec}>\operatorname{Re} d$, instead of (3.11), we have

$$
M(d, c, z)=-\frac{\Gamma(c) \Gamma(1-d)}{2 \pi i \Gamma(c-d)} \int_{\gamma_{2}}(-u)^{d-1}(1-u)^{c-d-1} e^{z u} \mathrm{~d} u,
$$

where $\gamma_{2}$ is the integration path given in (2.8). This integral representation can be established in the same manner as (2.8) or (2.5). Thus, with $d=a N+1(a<0), c=1$ and $z=\eta N, \mathbf{M}(d, c, z):=M(d, c, z) / \Gamma(c)$ can be written as

$$
\mathbf{M}(a N+1,1, \eta N)=\frac{1}{2 \pi i} \int_{\gamma_{2}} e^{N \widehat{\psi}(u)} \frac{\mathrm{d} u}{u-1},
$$

where $\widehat{\psi}(u)=a \ln (-u)-a \ln (1-u)+\eta u$. The saddle points $u_{ \pm}$of $\widehat{\psi}(u)$ are the same as those of $\psi(u)$ given in (3.14)

$$
u_{ \pm}=\frac{\eta \pm \sqrt{\eta^{2}+4 a \eta}}{2 \eta}
$$

It can be shown that the movements of $u_{ \pm}$when $a<0$ and $\eta<0$ are like those of the saddle points $w_{\mp}$ of $\widetilde{f}\left(t_{0}(w), w\right)$ when $a<0$. Also, we have

$$
\mathbf{M}^{\prime}(a N+1,1, \eta N)=\frac{1}{2 \pi i} \int_{\gamma_{2}} e^{N \widehat{\psi}(u)} \frac{u}{u-1} \mathrm{~d} u,
$$


where the derivative is taken with respect to the third variable in $\mathbf{M}(d, c, z)$.

Now, define the mapping $w \rightarrow u$ by

$$
\widetilde{f}\left(t_{0}(w), w\right)=\widehat{\psi}(u)+\gamma=a \ln (-u)-a \ln (1-u)+\eta u+\gamma
$$

with

$$
u\left(w_{+}^{( \pm)}\right)=u_{-}^{( \pm)}, \quad u\left(w_{-}\right)=u_{+},
$$

where $\eta$ and $\gamma$ are real numbers. (Note that, similar to (3.17a and (3.17b), since $w_{+}$is real and $w_{+}>1$, we have $w_{+}$on both upper and lower edges of the branch cut $[0,+\infty)$ along the real line. We denote these points by $w_{+}^{( \pm)}$, respectively. The situation is the same with $\left.u_{-}^{( \pm)}\right)$. Coupling (9.13) and (3.23), the double integral representation for $t_{n}(x, N+1)$ takes the canonical form

$$
\begin{aligned}
t_{n}(x, N+1)= & \frac{(-1)^{n}}{2 \pi i} \frac{\Gamma(n+N+2) e^{N \gamma}}{\Gamma(n+1) \Gamma(N-n+1)} \\
& \times \int_{-\infty}^{+\infty} \int_{\gamma_{u}} \frac{h(u, \tau)}{u-1} e^{N \widehat{\psi}(u)-N \tau^{2}} \mathrm{~d} u \mathrm{~d} \tau,
\end{aligned}
$$

instead of the one given in (3.29). In (9.15),

$$
h(u, \tau)=\frac{u-1}{w-1} \frac{\mathrm{d} w}{\mathrm{~d} u} \frac{\mathrm{d} t}{\mathrm{~d} \tau},
$$

and $\gamma_{u}$ is the steepest descent path of $\widehat{\psi}(u)$ passing through $u_{+}$. Let $h_{0}(u, \tau) \equiv h(u, \tau)$, and define the sequence $\left\{h_{n}(u, \tau)\right\}$ as in (6.9) - (6.11). Following the same argument as in Case $1\left(0 \leq a \leq \frac{1}{2}\right)$ of $₫ 6$, we obtain

$$
\begin{aligned}
t_{n}(x, N+1) \sim \frac{(-1)^{n} \Gamma(n+N+2) e^{N \gamma}}{\Gamma(n+1) \Gamma(N-n+1)}\left[\mathbf{M}(a N+1,1, \eta N) \sum_{l=0}^{\infty} \frac{c_{l}}{N^{l+\frac{1}{2}}}\right. \\
\left.+\mathbf{M}^{\prime}(a N+1,1, \eta N) \sum_{l=0}^{\infty} \frac{d_{l}}{N^{l+\frac{1}{2}}}\right]
\end{aligned}
$$

where $c_{l}$ and $d_{l}$ are as given in (6.13) and (6.14), respectively.

Although the constants $\eta$ and $\gamma$ in (9.17) and (8.2) are determined by two different sets of equations, namely, (9.13)-(9.14) and (3.15)-(3.16), respectively, one can use a single system of equations to determine these two constants for $a \in(-\infty, \infty)$. Indeed, we can define them by

$$
\left\{\begin{array}{l}
\operatorname{Re} f\left(t_{0}\left(w_{-}\right), w_{-}\right)=\operatorname{Re} \psi\left(u_{+}\right)+\gamma, \\
\operatorname{Re} f\left(t_{0}\left(w_{+}\right), w_{+}\right)=\operatorname{Re} \psi\left(u_{-}\right)+\gamma
\end{array}\right.
$$

where $f\left(t_{0}(w), w\right)$ and $\psi(u)$ are the same as those given in (3.15). (If $w_{-}$and $u_{+}$, or $w_{+}$and $u_{-}$, are on the branch cut, we should choose the correspoinding saddle points either both on the upper edge or both on the lower edge of the real line in the equations of (9.18) ). Thus, (9.17) and (8.2) are exactly the same, including the coefficients in these expansions, and the region of validity of expansion (8.2) has been extended from $0 \leq a \leq \frac{1}{2}$ to $-\infty<a \leq \frac{1}{2}$. 


\section{SMALL AND LARGE ZEROS}

It is well-known that from an asymptotic approximation of a function, one can deduce an asymptotic approximation for its zeros. The easiest way to achieve this is to apply the following result given in Hethcote [23]:

Lemma 2. In the interval $[a-\rho, a+\rho]$, suppose $f(t)=g(t)+\varepsilon(t)$, where $f(t)$ is continuous, $g(t)$ is differentiable, $g(a)=0, m=\min \left|g^{\prime}(t)\right|>0$, and

$$
E=\max |\varepsilon(t)|<\min \{|g(a-\rho)|,|g(a+\rho)|\} .
$$

Then, there exists a zero $c$ of $f(t)$ in the interval such that

$$
|c-a| \leq \frac{E}{m}
$$

To state our result, we first recall the numbers $a_{ \pm}$given in (3.3)

$$
a_{ \pm}=\frac{1 \pm \sqrt{1-b^{2}}}{2}
$$

where $b$ is a fixed number in $(0,1)$. Clearly, $0 \leq a_{-}<\frac{1}{2}<a_{+}<1$. The number $b$ can be as close to 0 or 1 as it may be, but it is fixed. Hence, $a_{-}$can be close to 0 but does not tend to 0 . Similarly, $a_{+}$can be close to 1 but does not approach 1 .

Theorem 4. Let $x_{N+1, s}$ denote the sth zero of the discrete Chebyshev polynomial $t_{n}(x, N+$ 1 ), arranged in ascending order $0<x_{N+1,1}<x_{N+1,2}<\cdots<x_{N+1, n-1}<x_{N+1, n}$.

If $s-1<a_{-} N$, then we have

$$
x_{N+1, s}=(s-1)+O\left\{e^{N\left[\widehat{\psi}\left(u_{-}\right)-\widehat{\psi}\left(u_{+}\right)\right]}\right\}
$$

as $N \rightarrow \infty$, where

$$
\widehat{\psi}\left(u_{-}\right)-\widehat{\psi}\left(u_{+}\right)=2 a \ln \frac{1+\sqrt{1+4 a / \eta}}{1-\sqrt{1+4 a / \eta}}+\eta \sqrt{1+4 a / \eta} .
$$

If $s>n-\left(1-a_{+}\right) N$, then we have

$$
\begin{aligned}
x_{N+1, s} & =N-x_{N+1, n-s+1} \\
& =N-n+s+O\left\{e^{N\left[\widehat{\psi}\left(u_{-}\right)-\widehat{\psi}\left(u_{+}\right)\right]}\right\}
\end{aligned}
$$

as $N \rightarrow \infty$.

Proof. The proof is quite straightforward. For $a<0$, we know from (6.21) that the discrete Chebyshev polynomials do not have zeros. For $0 \leq a<a_{-}$, it follows from (7.13), (7.14) that both $\mathbf{M}$ and $\mathbf{M}^{\prime}$ have zeros at approximately $x=m$ with a exponentially small error, where $m$ is a nonnegative integer; see the remark following (7.13). The results now are obtained by a direct application of Lemma 2 and the symmetry relation (1.8). Also, note that for $0 \leq a<a_{-}$, the exponent $N\left[\widehat{\psi}\left(u_{-}\right)-\widehat{\psi}\left(u_{+}\right)\right]$in (10.1) is negative, unless $\sqrt{1+4 a / \eta} \rightarrow 0$ in which case $a \rightarrow a_{-}$(i.e., $x$ is no longer small). 


\section{References}

[1] G. Szegö, Orthogonal Polynomials, 4th ed., American Mathematical Society, Providence, RI, 1975.

[2] R. Beals and R. Wong, Special Functions, A Graduate Text, Cambridge University Press, Cambridge, 2010.

[3] T.S.Chihara, An Introduction to Orthogonal Polynomials, Gordon and Breach, New York, 1978.

[4] F. B. Hildebrand, Introduction to Numerical Analysis, 2nd ed., McGraw-Hill, New York, 1974.

[5] N. Morrison, Introduction to Sequential Smoothing and Prediction, McGraw-Hill, New York, 1969.

[6] W. M. Y. Goh, Plancherel-Rotach asymptotics for the Charlier polynomials, Constr. Approx. 14 (1998), 151-168.

[7] Bo, Rui and R. Wong, Uniform asymptotic expansion of Charlier polynomials. Methods and Applications of Analysis 1 (1994), 294-313.

[8] X.-S. Jin and R. Wong, Uniform asymptotic expansions for Meixner polynomials, Constr. Approx. 14 (1998), 113-150.

[9] X.-S. Wang and R. Wong, Global asymptotics of the Meixner polynomials, Asymptotic Analysis, to appear.

[10] W.-Y. Qiu and R. Wong, Asymptotic expansion of the Krawtchouk polynomials and their zeros, Computational Methods and Function Theory 4 (2004), 189-226.

[11] D. Dai and R. Wong, Global asymptotics of Krawtchouk polynomials - a RiemannHilbert approach. Chin. Ann. Math. Ser. B 28 (2007), 1-34.

[12] W. M. Y. Goh and J. Wimp, On the asymptotics of the Tricomi-Carlitz polynomials and their zero distribution, SIAM J. Math. Anal. 25 (1994), 420-428.

[13] K. F. Lee and R. Wong, Uniform asymptotic expansions of the Tricomi-Carlitz polynomials. Proc. Amer. Math. Soc. 138 (2010), 2513-2519.

[14] J. Baik, T. Kriecherbauer, K. McLaughlin and P. K. Miller, Discrete Orthogonal Polynomials: Asymptotics and Applications, Princeton University Press, Princeton, NJ, 2007.

[15] S. Karlin and J. L. McGregor, The Hahn polynomials, formulas and an application, Scripta Mathematica 26 (1961), 33-46.

[16] F. W. J. Olver, D. W. Lozier, C. W. Clark and R. F. Boisvert, NIST Handbook of Mathematical Functions, Cambridge University Press, Cambridge, 2010. 
[17] M. V. Fedoryuk, Asymptotic methods in analysis, in Analysis I, Encyclopaedia of Mathematical Sciences, Volume 13, R. V. Gamkrelidze, ed., Springer-Verlag, Berlin Heidelberg 1989, 83-191.

[18] D. Kaminski, On the n-variable saddle point and steepest descent methods, in Asymptotic and Computational Analysis, Lecture Notes in Pure and Appl. Math. Series, No. 124, R. Wong, ed., Marcel Dekker, New York, 1990, 627-637.

[19] F. W. J. Olver, Asymptotics and Special Functions, Academic Press, New York, 1974. (Reprinted by A. K. Peters, Wellesley, MA, 1997.)

[20] R. Wong, Lecture Notes on Applied Analysis, World Scientific, Singapore, 2010.

[21] R. Wong, Asymptotic Approximations of Integrals, Academic Press, Boston, 1989. (Reprinted by SIAM, Philadelphia, PA, 2001)

[22] A. B. Olde Daalhuis and N. M. Temme, Uniform Airy-type expansions of integrals, SIAM J. Math. Anal. 25 (1994), 304-321.

[23] H. W. Hethcote, Error bounds for asymptotic approximations of zeros of transcendental functions, SIAM J. Math. Anal. 1 (1970), 147-152. 